\title{
Peculiarities of anharmonic lattice dynamics and thermodynamics of alkaline-earth metals
}

\author{
M.I.Katsnelson \\ Institute of Metal Physics, Ekaterinburg 620219, Russia \\ A. V. Trefilov, M. N. Khlopkin, and K. Yu. Khromov \\ Russian Research Centre "Kurchatov" Institute, Moscow 123182, Russia
}

\begin{abstract}
The calculations are performed for a broad range of the properties of $\mathrm{Ca}$ and $\mathrm{Sr}$ in the fcc and bcc phases. A detailed information on the magnitude and character of temperature dependence of anharmonic effects in the lattice dynamics over the entire Brillouin zone (frequency shifts and phonon damping, Gruneisen parameters) is given. A detailed comparison of the computational results for the heat capacity and thermal expansion with the experimental data is carried out; the theoretical results are in good agreement with the experiment.
\end{abstract}

63.20-e, 63.20.Ry 


\section{INTRODUCTION}

The investigation of anharmonic effects (AE) in lattice dynamics is a classical problem of solid-state physics. It is important, particularly, because of the role these effects can play in phenomena associated with structural phase transitions and melting in crystals (see, e.g.e. 3 ). At the same time, obtaining any information about the magnitude and scale of AEs from experiment and theory is a difficult problem. The experimental study of such "basic" AEs as the frequency shift and damping of phonons is very difficult and leads to a large uncertainty in the results (see, e.g., the data presented in 45 for bcc and fcc metals, respectively). Up to now first-principles microscopic calculations of AEs have been performed for one point of the Brillouin zone $(\mathrm{N})$ in the bcc phase of $\mathrm{Zr}$ and four points $(\mathrm{N}, \mathrm{P}, \omega, \mathrm{G})$ in Mol. Detailed information about AEs in the entire Brillouin zone and their temperature dependence has been obtained in 4 on the basis of pseudopotential theory for the bec phases of alkali and alkaline-earth metals. For these metals the most striking manifestations of AEs are due to the "soft-mode behavior" (the anomalous temperature dependence of the phonon frequencies) of the $\Sigma_{4}$ branch. It is of interest to calculate AEs for the "general position", i.e. for crystals which do not possess soft vibrational modes. Such crystals include most metals with closepacked structures, for example, fcc. In a recent paper 8 the results of calculations performed for the anharmonic effects in lattice dynamics of Ir having the fcc structure have been presented. In order to understand the specific features of AEs in lattice dynamics, related to structural phase transitions, it is interesting to investigate them in several phases for polymorphic metals. A classical example of structural phase transitions in metals is the fcc-bcc temperature transition in $\mathrm{Ca}$ and $\mathrm{Sr}$. Therefore studies of AE features in these metals seem to be important. In the present paper the $\mathrm{AE}$ features in $\mathrm{Ca}$ and $\mathrm{Sr}$ lattice dynamics and thermodynamics have been investigated basing on microscopic calculations of the heat capacity, thermal expansion, temperature frequency shifts, damping of phonons and Gruneisen parameters.

\section{APPROXIMATIONS AND COMPUTATION PROCEDURE}

As noted in Introduction, at present, ab initio calculations of phonon frequency with regard to AE have been only done for some highly symmetric points of the Brillouin zone in a few metals $\mathbf{6}$. Consistent $a b$ initio calculations of such quantities as phonon damping, as well as thermal expansion coefficients and other integral anharmonic effects in metals

are now extremely difficult. Therefore in works reported so far 1 . of interatomic interactions were used. For example, for alkali metals 2.6 and $\mathrm{Ir}^{8}$ the pair interaction approximation basing on pseudopotential theory was used, which gives a reliable description of a variety of lattice properties of these metals. Construction of a similar model for alkaline-earth metals appears to be difficult because of the specific features of electron structure. The fcc phase of $\mathrm{Ca}$ and $\mathrm{Sr}$ has many van Hove singularities in the density of electron states near the Fermi level $E_{F}$ and, therefore, for the adequate description of lattice properties allowance for related contributions to the total energy is important 1 . At the same time, the Fermi surface for these metals is close to that in the approximation of almost free electrons, and parameter $\left|V_{\boldsymbol{g}}\right| / E_{F}$ (where $\mathbf{g}$ is the reciprocal lattice vector, $\left|V_{\boldsymbol{g}}\right|$ is the 
Fourier component of pseudopotential) is small (less than 0.1) 10. As shown in 11 , under these conditions the proximity of the Fermi surface to the Brillouin zone boundary essentially contributes to the elastic moduli and to frequencies of long-wave phonons with the wave vector

$$
q \lesssim q_{c}=g_{1} \sqrt{\frac{\left|V_{\boldsymbol{g}}\right|\left|E_{c}-E_{F}\right|}{E_{F}}}
$$

where $E_{c}$ is the van-Hove singularity nearest to $E_{F}, g_{1}$ is the minimum vector of reciprocal lattice while the corresponding anomalous contributions to frequencies of phonons with $q>$ $q_{c}$ are much weaker. Therefore it may be expected that for the description of thermodynamics of fcc phase of $\mathrm{Ca}$ and $\mathrm{Sr}$ for $T \gtrsim \Theta_{D} q_{c} / g_{1} \approx 0.1 \Theta_{D}$ (where $\Theta_{D}$ is the Debye temperature) the ordinary approximation of pairwise interactions corresponding to the second order to perturbation theory for $\left|V_{\boldsymbol{g}}\right| / E_{F}$ would be applicable. This model was successfully used int for the description of the phonon spectra of $\mathrm{Ca}$ and $\mathrm{Sr}$ in the high temperature bcc phase.

In the present paper the phonon spectra, thermodynamic and anharmonic properties were calculated using the model corresponding to the second order of perturbation theory for pseudopotential (see, e.g.4.12). For the latter the Animalou-Heine expression

$$
V(q)=-\frac{4 \pi Z e^{2}}{q^{2}}\left[\cos q r_{0}+U\left(\frac{\sin q r_{0}}{q r_{0}}-\cos q r_{0}\right)\right] \exp \left[-0.03\left(\frac{q}{2 k_{F_{0}}}\right)^{4}\right]
$$

was used, where $Z=2$ is the ion charge, $e$ is the electron charge, $r_{0}, \mathrm{U}$ are the pseudopotential parameters listed in table $\mathbb{Q}, k_{F_{0}}$ is the Fermi momentum at zero pressure. For the screening the Geldart-Taylor approximation 13 with the Ceperley-Alder expression for correlation energy was used (see 12 for details). To eliminate the influence of fcc band structure peculiarities (the closeness of the Fermi surface to the bounadaries of the Brillouin zone), parameters $r_{0}$ and $U$ were fitted to the properties of high-temperature bcc phase: $\Omega=\Omega_{\text {exp }}\left(T=T_{s}\right)$ and $\Theta_{D}=\Theta_{D}^{\exp }\left(T=T_{s}\right)$ for $\operatorname{Sr}$ and $\Omega=\Omega_{\exp }\left(T=T_{s}\right)$ and $C^{\prime}=C_{\text {exp }}^{\prime}$ for $\mathrm{Ca}$. Here $T_{s}$ is the temperature of bcc-fcc transition (see table (1)), index "exp" indicates the related experimental value, $C^{\prime}=1 / 2\left(C_{11}-C_{12}\right)$ is one of the shear moduli.

For the AE calculations the standard anharmonic perturbation theory was used 14. W. With an accuracy up to terms of $\varkappa^{2}$ order, where $\varkappa=(m / M)^{1 / 4}$ is the adiabatic parameter, $m$, $M$ are the masses of electron and ion, respectively, the Hamiltonian of phonon subsystem can be written as:

$$
H=H_{0}+H_{q h}+H_{3}+H_{4}
$$

where

$$
H_{0}=\sum_{\lambda} \omega_{\lambda} b_{\lambda}^{+} b_{\lambda}
$$

is the Hamiltonian of harmonic approximation, $\lambda \equiv \boldsymbol{q} \xi$, q is the wave vector of phonon, $\xi$ is the number of phonon branch, $b_{\lambda}^{+}, b_{\lambda}$ are the phonon operators of creation and annihilation.

$$
H_{q h}=\sum_{\lambda i}\left(\frac{\partial \omega_{\lambda}}{\partial u_{i}}\right)_{u_{i}=0} u_{i} b_{\lambda}^{+} b_{\lambda}
$$


is the quasiharmonic Hamiltonian, $u_{i}$ are the deformation parameters (in cubic crystals it is sufficient to allow for dilatation $u_{1}, d u_{1}=d \ln \Omega$, where $\Omega$ is the lattice volume)

$$
\begin{gathered}
H_{3}=\sum_{\lambda_{1} \lambda_{2} \lambda_{3}} V^{(3)}\left(\lambda_{1}, \lambda_{2}, \lambda_{3}\right) Q_{\lambda_{1}} Q_{\lambda_{2}} Q_{\lambda_{3}} \\
=\sum_{\lambda_{1} \lambda_{2} \lambda_{3}} \Phi^{(3)}\left(\lambda_{1}, \lambda_{2}, \lambda_{3}\right) A_{\lambda_{1}} A_{\lambda_{2}} A_{\lambda_{3}} \\
H_{4}=\sum_{\lambda_{1} \lambda_{2} \lambda_{3} \lambda_{4}} V^{(4)}\left(\lambda_{1}, \lambda_{2}, \lambda_{3}, \lambda_{4}\right) Q_{\lambda_{1}} Q_{\lambda_{2}} Q_{\lambda_{3}} Q_{\lambda_{4}} \\
=\sum_{\lambda_{1} \lambda_{2} \lambda_{3} \lambda_{4}} \Phi^{(4)}\left(\lambda_{1}, \lambda_{2}, \lambda_{3}, \lambda_{4}\right) A_{\lambda_{1}} A_{\lambda_{2}} A_{\lambda_{3}} A_{\lambda_{4}}
\end{gathered}
$$

are the Hamiltonians of three- and four- phonon processes, respectively,

$$
Q_{\lambda}=\frac{1}{\sqrt{2 M \omega_{\lambda}}} A_{\lambda}, \quad A_{\lambda}=b_{\lambda}+b_{-\lambda}^{+}
$$

is the operator of phonon coordinate, $-\lambda \equiv-\mathbf{q}, \xi$. Here and below we put the Planck constant as $\hbar=1$. Expressions for the amplitudes of three- and four-phonon processes $\Phi^{(3)}, \Phi^{(4)}$, in terms of the pseudopotential model used are shown in 1 . With an accuracy up to terms of $\varkappa^{2}$ order we have the following expressions for anharmonic shifts of phonon frequencies and damping (see Appendix):

$$
\begin{gathered}
\Delta_{\lambda}=\Delta_{\lambda}^{(q h)}+\Delta_{\lambda}^{(3)}+\Delta_{\lambda}^{(4)} \\
\Delta_{\lambda}^{(q h)}=-\gamma_{\lambda} \frac{\Delta \Omega}{\Omega} \\
\Delta_{\lambda}^{(3)}=-18 \sum_{\lambda_{2} \lambda_{3}}\left|\Phi^{(3)}\left(\lambda,-\lambda_{2},-\lambda_{3}\right)\right|^{2}\left\{( 1 + N _ { \lambda _ { 2 } } + N _ { \lambda _ { 3 } } ) \left[\frac{1}{\omega_{\lambda}+\omega_{\lambda_{2}}+\omega_{\lambda_{3}}}\right.\right. \\
\left.\left.+\frac{\mathcal{P}}{\omega_{\lambda_{2}}+\omega_{\lambda_{3}}-\omega_{\lambda}}\right]+\left(N_{\lambda_{2}}-N_{\lambda_{3}}\right)\left[\frac{\mathcal{P}}{\omega_{\lambda_{3}}-\omega_{\lambda_{2}}-\omega_{\lambda}}-\frac{\mathcal{P}}{\omega_{\lambda_{2}}-\omega_{\lambda_{3}}-\omega_{\lambda}}\right]\right\} \\
\Delta_{\lambda}^{(4)}=12 \sum_{\lambda^{\prime}} \Phi^{(4)}\left(-\lambda, \lambda, \lambda^{\prime},-\lambda^{\prime}\right)\left(1+2 N_{\lambda^{\prime}}\right) \\
\left.+\left(\sum_{\lambda_{2} \lambda_{3}}-N_{\lambda_{3}}\right)\left[\delta\left(\omega_{\lambda_{3}}-\omega_{\lambda_{2}}-\omega_{\lambda}\right)-\delta\left(\omega_{\lambda_{2}}-\omega_{\lambda_{3}}-\omega_{\lambda}\right)\right]\right\}
\end{gathered}
$$

here 


$$
\gamma_{\lambda}=-\frac{\partial \ln \omega_{\lambda}}{\partial \ln \Omega}
$$

are the Gruneisen parameters, $\Delta \Omega$ is the change in the crystal volume due to the thermal expansion

$$
N_{\lambda}=\frac{1}{\exp \left(\omega_{\lambda} / T\right)-1}
$$

is the Planck distribution function, $\mathcal{P}$ is the symbol of principle value. In the particular calculations the approximation

$$
\begin{gathered}
\mathcal{P} \frac{1}{x} \approx \frac{x}{x^{2}+\varepsilon^{2}} \\
\delta(x) \approx \frac{1}{\pi} \frac{\varepsilon}{x^{2}+\varepsilon^{2}}
\end{gathered}
$$

was used for different small positive $\varepsilon$ with subsequent extrapolation $\varepsilon \rightarrow 0$. The details of the calculations, in particular, the summing up over the Brillouin zone is discussed in detail int.

\section{CALCULATIONS OF PHONON SPECTRA IN HARMONIC APPROXIMATION}

The results of calculations of phonon spectra and Gruneisen parameters (14) for fcc and bcc phases of $\mathrm{Ca}$ and $\mathrm{Sr}$ are shown in Figs. 115. As noted above, the theoretical model used is unapplicable for the description of phonon spectra in the fcc phase of these metals in the nearest vicinity of point $\Gamma$ because of unaccounted effects of Fermi surface proximity to the Brillouin zone boundaries 1 . It follows from Fig. 1 that $q_{c} \mathrm{c}$ from Eq. (1) is of the order of $0.1 g_{1}$. Fig. 2 shows that the phonon spectrum in the bcc phase of $\mathrm{Ca}$ and $\mathrm{Sr}$ is qualitatively similar to phonon spectra in the bcc phase of alkali metals characteristic features: presence of "soft" $\Sigma_{4}$ branch and prominent minimum for branch $\mathrm{F}_{1}$. In the fcc phase of these metals there are no soft mode anomalies. Fig. 3 displays such phonon spectra of bcc $\mathrm{Sr}$ for $T=T_{s}$ with allowance for anharmonic frequency shifts and corresponding experimental data in accordance with 15 . It is seen that there is a reasonable agreement between the calculated phonon spectra and experimental data. Unfortunately, any direct experimental data for $\omega(\mathbf{q})$ in the fcc phase of $\mathrm{Ca}$ and $\mathrm{Sr}$ are unavailable. The comparison of theory with the experiment for this phase will be done in the next Section basing on the data on temperature dependence of the lattice heat capacity. As seen from Figs. 4, 5 the Gruneisen model $\gamma_{\lambda}=$ const is absolutely unapplicable, even qualitatively, for the description of bcc phases in alkali metals. In the fcc phase the q-dependence is much weaker (provided that the vicinity of point $\Gamma$, where the model itself becomes unapplicable, is not considered). This seems to be because of lack of "soft modes" in the phonon spectra of fcc phase. 


\section{ANHARMONIC EFFECTS IN LATTICE DYNAMICS}

The calculation results for the anharmonic effects in the lattice dynamics for $\mathrm{Ca}$ and $\mathrm{Sr}$ are given in Figs. 6-15. The temperature dependencies of the frequency shift and phonon damping are shown for Ca as an example (Figs. 6, 7, 12, 13); for Sr the anharmonic effects are somewhat stronger than for Ca, nevertheless all dependencies are similar.

Figs. 6, 7 displays temperature dependencies of phonon frequency shifts:

$$
\begin{aligned}
\Delta \omega(T) & =\omega(T)-\omega(T=0) \\
& =\Delta_{\lambda}^{(q h *)}+\Delta_{\lambda}^{(3 *)}+\Delta_{\lambda}^{(4 *)}
\end{aligned}
$$

Here $\Delta_{\lambda}^{(q h *)}, \Delta_{\lambda}^{(3 *)}, \Delta_{\lambda}^{(4 *)}$ are values determined by Eqs. 10-13 respectively without the contribution of zero point oscillations, since such contributions enter both $\omega(T)$ and $\omega(T=0)$ and are compensated in the difference (18). Note also that since fitting of the pseudopotential papameters was done at the volume $\Omega=\Omega_{\text {exp }}\left(T=T_{s}\right.$ ) (see Section II), $\Delta \Omega$ entering right hand side of Eq. (10) is determined by the following relation: $\Delta \Omega=\Omega(T)-\Omega\left(T_{s}\right)$

It is seen that both in the bcc and in fcc phases $\Delta \omega(T)$ early (at $T \approx 0.1 \div 0.2 T_{p l}$ ) comes to high-temperature behavior $\Delta \omega(T) \sim T$. In the bcc phases of Ca and Sr the typical soft-mode behavior $d \omega / d T>0$ for $\Sigma_{4}$ branch takes place (see the data for $N_{4}$ in Fig. 7). As pointed out int the anharmonic effects in the bcc phases of alkaline-earth and alkaline metals are qualitatively similar in the whole, but, however, the scale of these effects in alkaline-earth metals is essentially larger. In the fcc phase of Ca and Sr there are no "soft modes" and $d \omega / d T<0$ for all vibrational modes.

Figs. 8, 9 shows the q-dependence of the relative frequency shift in $\mathrm{Ca}$ and $\mathrm{Sr}$. The comparison of the results for the fcc phase (Fig. 8) with those obtained in 8 for Ir indicates that the positions of frequency shift minima and maxima in the Brillouin zone depends essentially on the ion charge; in Ir at $Z=4.5$ maxima of $\delta$ are reached at symmetric points $\mathrm{X}$ and L, while in alkaline-earth metals they are significantly shifted. Moreover, in contrast with the temperature dependencies $\delta(T)$, there is a noticeable difference in the behavior of $\delta(\boldsymbol{q})$ in $\mathrm{Ca}$ and $\mathrm{Sr}$ (cf., for example, the dependence in $\Gamma-L$ direction in Fig. 8).

For the bcc phases of $\mathrm{Ca}$ and, particularly, $\mathrm{Sr}, \delta(\mathbf{q})$ has a prominent maximum in the narrow region of $\mathbf{q}$-space near point $\mathrm{N}$ for the soft branch $\Sigma_{4}$ for which $\delta>0$ (see Fig. 9).

On example of $\mathrm{Ca}$, Figs. 10, 11] show the contributions of three- and four- phonon processes to the frequency shift. In both phases these contributions have opposite signs in the larger part of the Brillouin zone $\left(\Delta_{3}<0, \Delta_{4}>0\right)$ The mentioned above "soft mode" behavior of the branch $\Sigma_{4}$ in the bcc phase is due to a sharp increase in $\Delta_{4}$ as in alkali metals 1 and in $\mathrm{BaTiO}_{3}$-type ferroelectrics 1 .

In Figs. 12, 13 the temperature dependence of phonon damping in the symmetric points of Brillouin zone is shown for Ca as an example. First of all it should be pointed out that, in accordance with Eq. (13) the damping does not vanish even at $T=0$. It is due to three-phonon processes (phonon decay into two phonons) and has a smallness of $\varkappa^{2}$ order. It is seen from Figs. 12, 13 that for Ca it is of the order of $0.2 \div 0.5 \%$ of phonon frequency. As in the case of anharmonic frequency shift, it is seen that transition to asymptotic $\Gamma \sim T$ is reached very early (at $T \approx 0.1 T_{p l}$ ). 
Figs. 14, 15 show the q-dependence of relative damping of phonons $\eta$ in Ca and Sr. In the fcc phase $\eta$ has essentially nonmonotonous dependence on the wave vector, reaches values of about $25 \%$ in $\mathrm{Sr}$ and does not exceed $12 \%$ in Ca. In the bcc phase of Sr in the vicinity of point $\mathrm{N} \eta$ reaches about 1 for the soft mode $\Sigma_{4}$, which formally indicates inapplicability of the anharmonic perturbation theory in this narrow region. At the same time in the larger part of the Brillouin zone the anharmonic perturbation theory seems to be applicable. In view of this a problem arises on accurate separation of the contribution of the vicinity of point $\mathrm{N}$ to integral anharmonic characteristics such as free energy. This question will be considered elsewhere 16 .

\section{ANALYSIS OF EXPERIMENTAL DATA ON HEAT CAPACITY AND THERMAL EXPANSION}

\section{A. Heat capacity}

The lattice heat capacity is determined by the phonon state density $g(\omega)$. Some characteristics of the latter, which can be used for comparing our description of phonon spectra with the experiment can be found from the analysis of temperature dependence of heat capacity at the constant pressure $C_{p}(T)$.

Here we carried out the separation of the lattice components from the heat capacity measured in the experiment and determined some average frequencies (momenta) of phonon spectrum, without using any model.

In our analysis we used the following set of data: for $\mathrm{Sr}$ - direct experimental data from 17 , for $\mathrm{Ca}$ - the data from handbooks; in the region 2-50 $\mathrm{K}$ - the data from handbook 18 which in the region $2-10 \mathrm{~K}$ is based on 19 , and in the region $50-600$ - the data taken from handbook 20 .

In the analysis we neglected the temperature dependence of the electron heat capacity coefficient and assumed that the anharmonism is weak. The assumption on smallness of anharmonic contributions to the thermodynamical properties of metals is justified as it was checked by a direct calculation and found to be true even for alkali metals having soft phonon modes 3 . Under these assumptions the heat capacity measured experimentally at a constant pressure, $C_{p}$ is described by the relations:

$$
\begin{gathered}
C_{p}=C_{p h}+C_{a} \\
C_{a}=\left\{\gamma+(A-\gamma)\left(C_{p h} / 3 R\right)^{2}\right\} T
\end{gathered}
$$

where

$C_{p h}$ is the phonon component of the heat capacity in the harmonic approximation,

$C_{a}$ is the sum of contributions to the heat capacity, having the linear temperature dependence and coming from the anharmonic effects, thermal expansion of the lattice and conduction electrons;

$\gamma$ is the coefficient of electron heat capacity at low temperatures,

$A$ is the coefficient of linear in temperature term at high temperatures, 
$R$ is the gas constant.

The interpolation formula (20) for $C_{a}$ gives the corresponding linear temperature asymptotics both at low- and high-temperatures and ensures a smooth transition between the low and high temperature asymptotics in accordance with the law similar to Nernst-Linderman formula21.

In the high-temperature region the phonon component of heat capacity was described by the expression proposed by Naumov2 2

$$
\begin{gathered}
C_{p h}=3 R\left\{1-\frac{1}{12}\left(\frac{\Omega_{2}}{T}\right)^{2}+\frac{1}{240}\left(\frac{\Omega_{4}}{T}\right)^{4}+\varphi\left(\frac{\Omega_{*}}{T}\right)\right\} \\
\varphi(z)=\frac{z^{2} \exp (z)}{(1-\exp (z))^{2}}-\left(1-\frac{1}{12} z^{2}+\frac{1}{240} z^{4}\right)
\end{gathered}
$$

Here the asymptotic expansion of phonon heat capacity over small parameter $z=\Omega / T$ is used:

$$
C_{p h}=3 R\left(1-\sum_{n=2}^{\infty} \frac{(n-1) B_{n}}{n !}\left(\frac{\Omega_{n}}{T}\right)^{n}\right)
$$

where $B_{n}$ are the Bernulli numbers $\left(B_{2}=1 / 6, B_{4}=-1 / 30, B_{6} 6=1 / 42, B_{8}=-1 / 30\right.$, $B_{10}=5 / 66$ etc. For the odd $\mathrm{n}$, beginning from $\mathrm{n}=3$, all $\left.B_{n}=0\right)$.

In (21) the second and fourth order correction for $\Omega / T$ are separately written, and function $\varphi\left(\frac{\Omega_{*}}{T}\right)$ allows for all higher corrections in the "Einstein" approximation, i.e. assuming $\Omega_{n}=\Omega_{*}$ for all $n \geq 6$. The values $\Omega_{n}$ characterize momenta (mean frequencies) of phonon spectrum according to the relation:

$$
\left(\Omega_{n}\right)^{n}=<\omega^{n}>=\int_{0}^{\infty} g(\omega) \omega^{n} d \omega / \int_{0}^{\infty} g(\omega) d \omega
$$

The value $\gamma$ entering $C_{a}(20)$ was determined in the standard manner: by fitting of heat capacity in the low temperature region (particularly, $2-5 \mathrm{~K}$ for Ca and $5-15 \mathrm{~K}$ for $\mathrm{Sr}$ ) by the relation $C_{p}=\gamma T+\beta T^{3}$. The values $\gamma, \beta$ as well as the limiting low-temperature value of the Debye temperature $\Theta_{L T}$ related to $\beta$ by relation $\beta=12 \pi^{4} R /\left(5 \Theta_{L T}^{3}\right)$ are listed in Table [II. The estimations of $\Theta_{L T}$ we obtained from the set of data used reasonably agree with those available in the literature (see 17.19$)$.

The value $A$ together with $\Omega_{2}, \Omega_{4}$ and $\Omega_{*}$ were determined using the least square method by the fitting of heat capacity by relations (21, 22) in the temperature region 40-600 $\mathrm{K}$ for $\mathrm{Ca}$ and $32-350 \mathrm{~K}$ for $\mathrm{Sr}$. Within this temperature range these relations described the experimental results with least square deviation of the order of $1 \%$ for $\mathrm{Ca}$ and $0.3 \%$ for Sr. The parameters $A, \Omega_{2}, \Omega_{4}$ and $\Omega_{*}$ determined by the least square method are shown in Table II. The table also gives the limiting high-temperature value of Debye temperature $\Theta_{H T}=\Theta_{D}(T)$ where $\Theta_{L T} \geq T<T_{m}$ ( $T_{m}$ is the melting temperature) related to the second momentum of the phonon spectrum by: $\Omega_{2}=\Theta_{H T} \sqrt{3 / 523}$. 
It should be pointed out that the lower value of the least square deviation for Sr is due to that over the whole temperature range the experimental data from the same source were used. On the other hand, its higher value for Ca seems to be due to non-smooth joining of the data at the transition from one source to another one.

The analysis performed permitted the contribution of $C_{p h}$ in the harmonic approximation to be separated from the total heat capacity and the electron and anharmonic contributions to be left out. Some phonon spectrum momenta are expressed directly via phonon heat capacity integrals24.

$$
\begin{gathered}
<\omega>=2 \int_{0}^{\infty}\left(1-\frac{C_{p h}}{3 R}\right) d T \\
<\omega^{-1}>=\frac{3}{\pi^{2}} \int_{0}^{\infty} \frac{C_{p h}}{3 R} T^{-2} d T \\
<\omega^{-2}>=0.138651 \int_{0}^{\infty} \frac{C_{p h}}{3 R} T^{-3} d T \\
<\omega^{-1} \log \omega>=\frac{3}{\pi^{2}} \int_{0}^{\infty} \frac{C_{p h}}{3 R} \log \left(\frac{T}{0.70702}\right) T^{-2} d T
\end{gathered}
$$

We calculated these momenta with the integration over the experimental points in the temperature region 10-300 $\mathrm{K}$ and outside this region - over low temperature asymptotic

$C_{p h}=\beta T^{3}$ and high temperature asymptotic in the Debye spectrum model as in 25 . The mean frequencies corresponding to these momenta are listed in Table $\mathbb{1}$. The meanings of $\Omega_{-2}, \Omega_{-1}, \Omega_{1}$ corresponding to that of $\Omega_{n}$ in relation 24 for $\mathrm{n}=-2$, - 1 and 1 while the meaning of $\Omega_{l o g}$ is determined by relation:

$$
\begin{aligned}
\log \left(\Omega_{\text {log }}\right) & =\frac{<\omega^{-1} \log \omega>}{<\omega^{-1}>} \\
& =\int_{0}^{\infty} \frac{g(\omega) \log \omega}{\omega} d \omega / \int_{0}^{\infty} \frac{g(\omega)}{\omega} d \omega
\end{aligned}
$$

The contribution of $C_{p h}$ in the harmonic approximation separated from the total heat capacity is shown in Figs. 16, 17.

\section{B. Thermal expansion}

The coefficient of thermal expansion $\alpha(T)$ contains two contributions: the electron one $\alpha_{e}$ and the lattice one $\alpha_{l}$. In the low temperature region the electron contribution is linearly dependent on temperature, while the lattice contribution is proportional to $T^{3}$. This makes it possible to separate these contributions fitting the experimental data by the dependence: 


$$
\alpha(T)=D T+E T^{3}
$$

In the paper 26 the electron contribution to thermal expansion of $\mathrm{Ca}$ and $\mathrm{Sr}$ is determined. For the determination of the lattice contribution to thermal expansion we used the following set of experimental data: in the low temperature region - the data from 26 and in the high temperature region - the data from 27 for $\mathrm{Ca}$ and 28 for Sr. The phonon contribution to thermal expansion was determined as the difference of the experimental data and the electron contribution. It was assumed that the electron contribution is linear by temperature over the whole temperature region, its value being determined by low temperature asymptotic in 26. The lattice contribution to thermal expansion separated from the experimental data is shown in Figs. 18, 19.

The lattice contributions to the heat capacity and the coefficient of thermal expansion, separated from the experimental data were used for the determination of the Gruneisen lattice parameter by the formula:

$$
G=\frac{3 \alpha B V_{m}}{C_{p h}}
$$

where $B$ is the bulk modulus, $\alpha$ is the temperature coefficient of linear expansion, $V_{m}$ is the molar volume. The temperature dependence of the Gruneisen parameter $\gamma(T)$ is shown in Figs. 18, 19.

\section{CALCULATIONS OF HEAT CAPACITY AND THERMAL EXPANSION}

Figs. 16, 17 give the computation results of lattice heat capacity of $\mathrm{Ca}$ and $\mathrm{Sr}$ in the harmonic approximation and the effective Debye temperature. It is seen that, with the exception of the region of lowest temperatures where the phonon spectrum model itself becomes inapplicable (see Section III), the theory is in a perfect agreement with the experiment. It is especially well seen in Table [I], where the computation data are compared with the momenta of phonon density of states, found from the experimental data.

Figs. 18, 19 displays the computation results for the temperature dependence of the volume $\Delta \Omega(T)$ and of the Gruneisen parameter $\gamma(T)$ for Ca and Sr.It is related to the Gruneisen parameters by the following expression:

$$
\gamma(T)=\frac{\sum_{\lambda} \gamma_{\lambda} N_{\lambda}\left(1+N_{\lambda}\right) \omega_{\lambda}^{2}}{\sum_{\lambda} N_{\lambda}\left(1+N_{\lambda}\right) \omega_{\lambda}^{2}}
$$

One can see that the temperature dependence of the macroscopic Gruneisen parameter is a rather smooth. At the same time, our calculations show a strong temperature dependence of microscopic Gruneisen parameters for the soft branch in BCC phases of Ca and Sr (see Table [II). It would be very interesting to check this prediction experimentally. 


\section{CONCLUSIONS}

In the present paper a simple, and at the same time, adequate model of the interatomic interactions is proposed, which describes with a reasonably high accuracy the lattice properties of fcc and bcc phases of $\mathrm{Ca}$ and Sr. As a result, the dependence of AE in the dynamics of lattice on its geometry could be investigated. The comparison of the results obtained for the fcc phase of $\mathrm{Ca}$ and $\mathrm{Sr}$ with earlier results for $\mathrm{Ir}$ - demonstrates an essential dependence of $\mathrm{AE}$ on the character of itneratomic interactions for the given crystal structure. As the question on the AE scale, particularly, for metals with polymorphic transformation (in this case bcc-fcc) is one of the key problems in the lattice dynamics, performance of further experimental investigations is desirable.

\section{APPENDIX:}

Here we shall give a short derivation of equations for the temperature shift of frequencies and phonon damping (9-13). In contrast to the basing work 14 and subsequent publications, where the diagram method for Matsubara Green functions was used, we employ the method

of two time Green functions $A 1$. We shall begin with the chain of equations of motion for delayed two time Green functions

$$
\omega \ll A\left|B \gg_{\omega}=\langle[A, B]\rangle+\ll[A, H]\right| B \gg
$$

where

$$
\ll A\left|B \gg_{\omega}=-i \int_{0}^{\infty} d t \exp [i(\omega+i \varepsilon) t]\langle[A(t), B(0)]\rangle\right|_{\varepsilon \rightarrow+0}
$$

The bracketed expression indicates the commutator of operators, and that within the French quats - the averaging over the Gibbs ensemble. For the Hamiltonian (낙) we have the accurate equation:

$$
\begin{aligned}
\left(\omega-\omega_{\lambda}\right) \ll b_{\lambda} \mid b_{\lambda}^{+} \gg_{\omega}= & 1+3 \sum_{\lambda_{2} \lambda_{3}} \Phi^{(3)}\left(-\lambda, \lambda_{2}, \lambda_{3}\right) \ll A_{\lambda_{2}} A_{\lambda_{3}} \mid b_{\lambda}^{+} \gg_{\omega} \\
& +4 \sum_{\lambda_{2} \lambda_{3} \lambda_{4}} \Phi^{(4)}\left(-\lambda, \lambda_{2}, \lambda_{3}, \lambda_{4}\right) \ll A_{\lambda_{2}} A_{\lambda_{3}} A_{\lambda_{4}} \mid b_{\lambda}^{+} \gg_{\omega}
\end{aligned}
$$

With an accuracy up to $\varkappa^{2}$ the Green function describing the contribution of four-phonon processes to (A3) can be decoupled

$$
\begin{aligned}
\ll A_{\lambda_{2}} A_{\lambda_{3}} A_{\lambda_{4}} \mid b_{\lambda}^{+} \gg_{\omega}= & \left\langle A_{\lambda_{2}} A_{\lambda_{3}}\right\rangle \ll A_{\lambda_{4}}\left|b_{\lambda}^{+} \gg_{\omega}+\left\langle A_{\lambda_{2}} A_{\lambda_{4}}\right\rangle \ll A_{\lambda_{3}}\right| b_{\lambda}^{+} \gg_{\omega} \\
& +\left\langle A_{\lambda_{3}} A_{\lambda_{4}}\right\rangle \ll A_{\lambda_{2}} \mid b_{\lambda}^{+} \gg_{\omega} \\
= & \delta_{\lambda_{2} \lambda_{3}}\left(1+2 N_{\lambda_{2}}\right) \delta_{\lambda_{4} \lambda}+\delta_{\lambda_{2} \lambda_{4}}\left(1+2 N_{\lambda_{1}}\right) \delta_{\lambda_{3} \lambda} \\
& +\delta_{\lambda_{2} \lambda_{4}}\left(1+2 N_{\lambda_{3}}\right) \delta_{\lambda_{2} \lambda}+\ll b_{\lambda} \mid b_{\lambda}^{+} \gg_{\omega}
\end{aligned}
$$


This is immediately followed by the result (12) for the contribution of four-phonon processes to the frequency shift. For the determination of the contribution of three-phonon processes we must write the chain of equations for the Green-functions entering the following relation

$$
\begin{aligned}
\ll A_{\lambda_{2}} A_{\lambda_{3}} \mid b_{\lambda}^{+} \gg_{\omega}= & \ll b_{\lambda_{2}} b_{\lambda_{3}}\left|b_{\lambda}^{+} \gg_{\omega}+\ll b_{-\lambda_{2}} b_{\lambda_{3}}\right| b_{\lambda}^{+} \gg_{\omega} \\
& +\ll b_{\lambda_{2}} b_{-\lambda_{3}}\left|b_{\lambda}^{+} \gg_{\omega}+\ll b_{-\lambda_{2}} b_{-\lambda_{3}}\right| b_{\lambda}^{+} \gg_{\omega}
\end{aligned}
$$

and make there decoupling. Upon separation of the real and imaginary parts of Green function $\ll b_{\lambda} \mid b_{\lambda}^{+} \gg_{\omega}$ we obtain immediately the result (9-13). The quasiharmonic frequency shift proves to be then as expressed through the amplitudes of three-phonon processes:

$$
\Delta_{\lambda}^{(q h)}=-36 \sum_{\lambda_{2} \lambda_{3}} \frac{1+2 N_{\lambda_{3}}}{\omega_{\lambda_{2}}} \Phi\left(\lambda,-\lambda, \lambda_{2}\right) \Phi\left(\lambda_{3},-\lambda_{3},-\lambda_{2}\right)
$$

As far as we know this expression is new, although the relation itself of thermal expansion to three-phonon anharmonism was qualitatively discussed as early as in the classical book by Paierls $A 2$. 


\section{REFERENCES}

${ }^{1}$ V. G. Vaks, Introduction to the Microscopic Theory of Ferroelectrics. (Nauka, Moscow, 1973).

${ }^{2}$ V. G. Vaks, S. P. Kravchuk, and A. V. Trefilov, J. Phys. F10, 2325 (1980).

${ }^{3}$ M. I. Katsnelson and A. V. Trefilov, Phys. Metal. Metallogr. 64 (4), 1 (1987).

${ }^{4}$ V. G. Vaks, S. P. Kravchuk, and A. V. Trefilov, J. Phys. F10, 2105 (1980).

${ }^{5}$ M. Zoli, G. Santoro, V. Bortolani, A. A. Maradudin, and R. F. Wallis, Phys. Rev. B41, 7507 (1990).

${ }^{6}$ Y. Y. Ye, Y. Chen, K. M. Ho, B. N. Harmon, and P. A. Lindgard, Phys. Rev. Lett. 58, 1769 (1987); Y. Y. Ye, K. M. Ho, Y. Chen, and B. N. Harmon, J. Phys.: Cond. Matter 3, 9629 (1991).

${ }^{7}$ V. G. Vaks, G. D. Samolyuk, and A. V. Trefilov, Phys. Lett. A127, 37 (1988).

${ }^{8}$ M. I. Katsnelson, A. V. Trefilov, and K. Yu. Khromov, JETP Lett. 69, 688 (1999).

${ }^{9}$ A. O. E. Animalu, Intermediate Quantum Theory of Crystalline Solids (Prentice Hall, Englewoods Cliffs, 1977).

${ }^{10}$ M. I. Katsnelson, G. V. Peschanskikh, and A. V. Trefilov, Sov. Phys.: Solid State 32, 272 (1990).

${ }^{11}$ V. G. Vaks and A. V. Trefilov, J. Phys: Cond. Matter 3, 1389 (1991).

${ }^{12}$ S. V. Vonsovsky, M. I. Katsnelson, and A. V. Trefilov, Phys. Metal. Metallogr. 76, 247 (1993).

${ }^{13}$ D. J. Geldart and R. Taylor, Canad. J. Phys. 48, 155 (1970).

${ }^{14}$ R. A. Cowley, Adv. Phys. 12, 421 (1963).

15 J. Mizuki, C. Stassis, Phys. Rev. B32, 8372 (1985); ibid., B35 872 (1987).

${ }^{16}$ M. I. Katsnelson, A. F. Maksjutov, A. V. Trefilov, and K. Yu. Khromov (unpublished).

${ }^{17}$ Boerio J. and Westrum E.F., J. Chem. Thermodynamics 10, 1 (1978).

${ }^{18}$ R.Hultgren, R.D.Decai, D.T.Hawkins, M.Gleiser, K.K.Kelley, and D.D.Wagman, Selected values of the thermodynamic properties of the elements (Metals Park (Ohio), Amer. Soc. for Metals, 1973).

${ }^{19}$ L. M. Roberts, Proc. Phys. Soc. B70, 738 (1957).

${ }^{20} \mathrm{H}$. Landolt and R. Bornstein, Zahlenwerte und Funktionen aus Physik Chemie, Astronomie, Geophysik und Technik (Bd.2. T.4-6. Aufl. Berlin, Springer, 1960).

${ }^{21}$ L. A. Reznitskii, Calorimetry of Solids (Moscow, MSU, in Russian ,1981).

${ }^{22}$ V. N. Naumov, Phys. Rev. B49, 13247 (1994).

${ }^{23}$ A. A. Maradudin, E. W. Montroll, and G. H. Weiss. Theory of lattice dynamics in the harmonic approximation (Acad. Pres., N. Y., 1963).

${ }^{24}$ A. Junod, Solid State Communications 33, 55 (1980).

${ }^{25}$ A. V. Mirmelstein, A. E. Karkin, V. E. Arkhipov, and V. I. Voronin, Phys. Metal. Metallogr. 58, 1008 (1984).

${ }^{26}$ G.K.White, J.Phys. F2, 865.

27 S. I. Novikova, Thermal expansion of solids (Nauka, Moscow, 1974).

${ }^{28}$ N. S. Grigoriev and E. Z. Meilikhov, Physical values. The handbook. (Energoatomizdat, Moscow, 1991).

${ }^{A 1}$ D. N. Zubarev, Uspekhi Fiz. Nauk 71, 71 (1960).

${ }^{A 2}$ R. E. Peierls, Quantum Theory of Solids (Clarendon Press, Oxford,1995). 


\section{FIGURES}

FIG. 1. Phonon spectrum of fcc phase of $\mathrm{Ca}$ (solid line) and $\mathrm{Sr}$ (dashed line) in the harmonic approximation in units of ion plasma frequency $\omega_{p l}=\left(4 \pi Z e^{2} / M \Omega\right)^{1 / 2}$. Indices 1 and 2 denote longitudinal and transverse branches respectively.

FIG. 2. Phonon spectra for bcc phase of Ca (solid line) and Sr (dashed line) in units $\omega_{p l}$ in the harmonic approximation.

FIG. 3. Phonon spectra for bcc phase of $\operatorname{Sr}$ at $T=T_{s}$; solid line is the calculation (with the account for anharmonic shift, see below), points - the experimental data from 15 .

FIG. 4. Gruneisen parameters for fcc phase of Ca (solid line) and $\mathrm{Sr}$ (dashed line).

FIG. 5. Gruneisen parameters for bcc phase of Ca (solid line) and Sr (dashed line).

FIG. 6. Temperature dependence of frequency shift determined by Eq. (18) at symmetric points of the Brillouin zone for fcc Ca; $\omega_{p l}=\left(4 \pi Z e^{2} / M \Omega\right)^{1 / 2}$ is the plasma ion frequency, $T_{p l}$ is the corresponding temperature (for $\mathrm{Ca} T_{p l}=476 \mathrm{~K}$.) The symbols correspond to different vibration modes: $\circ-W_{1}, \triangle-W_{2}, \square-X_{1}, \diamond-X_{2}, \diamond-L_{1}, \star-L_{2}$.

FIG. 7. Temperature dependence of frequency shift at symmetric points of the Brillouin zone for bcc Ca; The symbols correspond to different vibration modes: $\circ-N_{1}, \diamond-N_{3} \triangle-N_{4}$, $-H, \varnothing-P$.

FIG. 8. Change of the relative frequency shift $\delta_{\xi \mathbf{k}}(T)=\Delta \omega_{\xi \mathbf{k}}(T) / \omega_{\xi \mathbf{k}}(0)$ in the Brillouin zone for fcc phases of $\mathrm{Ca}$ and $\mathrm{Sr}$ at the temperature of structure transition $T=T_{s}\left(T_{s}=726 \mathrm{~K}\right.$ for Ca and $930 \mathrm{~K}$ for $\mathrm{Sr}), \Delta \omega_{\xi \mathbf{k}}(T) /$ is determined by Eq. (18). Solid line - Ca, dashed line - Sr.

FIG. 9. The same as Fig. 8 for bcc phase of Ca and Sr.

FIG. 10. Change in the Brillouin zone of the anharmonic frequency shift determined by formulae (11), (12) for fcc Ca at $T=T_{s}$.

FIG. 11. The same as Fig. 10 for bcc phase of Ca.

FIG. 12. Temperature dependence of phonon damping at symmetric points of Brillouin zone for fcc Ca. $T_{p l}$ and the symbols are the same as in Fig. 6 . 
FIG. 13. The same as Fig. 12 for bcc phase of Ca. The symbols are the same as in Fig. 7 .

FIG. 14. Change in the Brillouin zone of relative damping $\eta_{\xi \mathbf{k}}(T)=\Gamma_{\xi \mathbf{k}}(T) / \omega_{\xi \mathbf{k}}(0)$ for fcc Ca and $\mathrm{Sr}$ at $T=T_{s}$. Solid line $-\mathrm{Ca}$, dashed line $-\mathrm{Sr}$.

FIG. 15. The same as Fig. 14 for bcc phase of Ca and Sr.

FIG. 16. Phonon contribution to the lattice heat capacity of $\mathrm{Ca}$; in the insert the temperature dependence of effective Debye temperature is shown. $\diamond$ is the result of experimental treatment (see the text); solid line - the calculation result for the bcc phase, dashed line - for the fcc phase.

FIG. 17. The same as Fig. 16 for Sr.

FIG. 18. Phonon contribution to the thermal expansion of $\mathrm{Ca}$; in the insert the temperature dependence of Gruneisen parameter is shown. The meaning of symbols and lines is the same as in Fig. 16.

FIG. 19. The same as Fig. 18 for Sr. 


\section{TABLES}

TABLE I. Model parameters.

\begin{tabular}{cccccccc}
\hline \hline Metal & $T_{s}$ & $\Omega_{\text {exp }}(\mathrm{au})$ & $\omega_{p l}(\mathrm{THz})$ & $T_{m}(\mathrm{~K})$ & $r_{0}(\mathrm{au})$ & $\mathrm{U}$ & $\mathrm{Z}$ \\
\hline $\mathrm{Ca}$ & 726 & 303.4 & 9.91 & 1112 & 2.545 & 0.636 & 2 \\
$\mathrm{Sr}$ & 930 & 394.6 & 5.88 & 1045 & 3.024 & 0.787 & 2 \\
\hline \hline
\end{tabular}

TABLE II. Phonon spectra momenta.

\begin{tabular}{|c|c|c|c|c|c|c|}
\hline \multirow[t]{3}{*}{ momenta } & \multicolumn{3}{|c|}{$\mathrm{Ca}$} & \multicolumn{3}{|c|}{$\mathrm{Sr}$} \\
\hline & experiment & theory & theory & experiment & theory & theory \\
\hline & FCC & FCC & $\mathrm{BCC}$ & FCC & FCC & $\mathrm{BCC}$ \\
\hline$\Omega_{l o g}, \mathrm{~K}$ & 136 & 138 & 128 & 81 & 78 & 67 \\
\hline$\Omega_{-2}, \mathrm{~K}$ & 130 & 142 & 121 & 77 & 77 & 64 \\
\hline$\Omega_{-1}, \mathrm{~K}$ & 152 & 158 & 148 & 89 & 88 & 80 \\
\hline$\Omega_{1}, \mathrm{~K}$ & 181 & 185 & 182 & 102 & 102 & 99 \\
\hline$\Omega_{2}, \mathrm{~K}$ & 195 & 193 & 193 & 107 & 107 & 106 \\
\hline$\Omega_{4}, \mathrm{~K}$ & 214 & 207 & 208 & 116 & 114 & 114 \\
\hline$\Omega_{*}, \mathrm{~K}$ & 220 & & & 120 & & \\
\hline$\gamma, \frac{m J}{m o l K^{2}}$ & 3 & & & 13.8 & & \\
\hline$\beta, \frac{m J}{m o l K^{4}}$ & $1.87 \times 10^{-2}$ & & & $8.9 \times 10^{-2}$ & & \\
\hline$\Theta_{L T}, \mathrm{~K}$ & 232 & & & 138 & & \\
\hline $\mathrm{A}, \frac{m J}{m o l K^{2}}$ & 9.1 & & & 6.9 & & \\
\hline$\Theta_{H T}, K$ & 252 & 249 & 249 & 139 & 138 & 137 \\
\hline
\end{tabular}

TABLE III. Temperature dependence of Gruneisen parameter.

\begin{tabular}{|c|c|c|c|c|c|}
\hline & $N_{1}$ & $N_{3}$ & $N_{4}$ & $\mathrm{H}$ & $\mathrm{P}$ \\
\hline \multicolumn{6}{|c|}{$\mathrm{Ca}, \mathrm{BCC} \mathrm{T}=726 \mathrm{~K}$} \\
\hline$\gamma_{0}$ & 1.60 & 1.12 & 3.60 & 1.31 & 1.66 \\
\hline$\Delta \gamma(T)$ & 0.059 & 0.12 & -2.87 & 0.24 & -0.13 \\
\hline$\gamma(T)$ & 1.66 & 1.24 & 0.73 & 1.55 & 1.53 \\
\hline \multicolumn{6}{|c|}{$\mathrm{Sr}, \mathrm{BCC} \mathrm{T}=930 \mathrm{~K}$} \\
\hline$\gamma_{0}$ & 1.69 & 1.16 & 5.44 & 1.37 & 1.77 \\
\hline$\Delta \gamma(T)$ & 0.13 & 0.31 & -20.0 & 0.33 & -0.37 \\
\hline$\gamma(T)$ & 1.82 & 1.47 & -14.6 & 1.7 & 1.40 \\
\hline
\end{tabular}


fig. 1

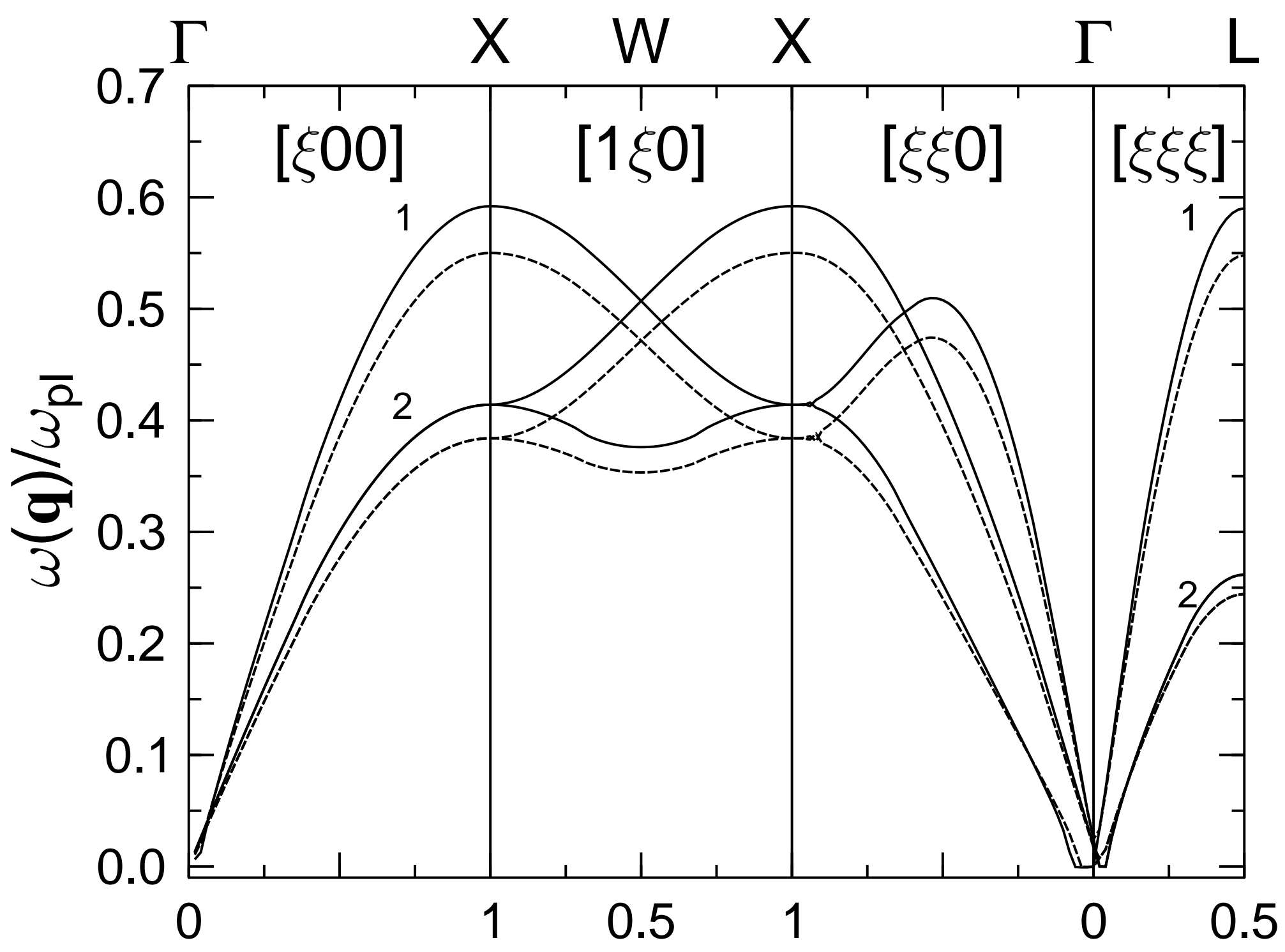



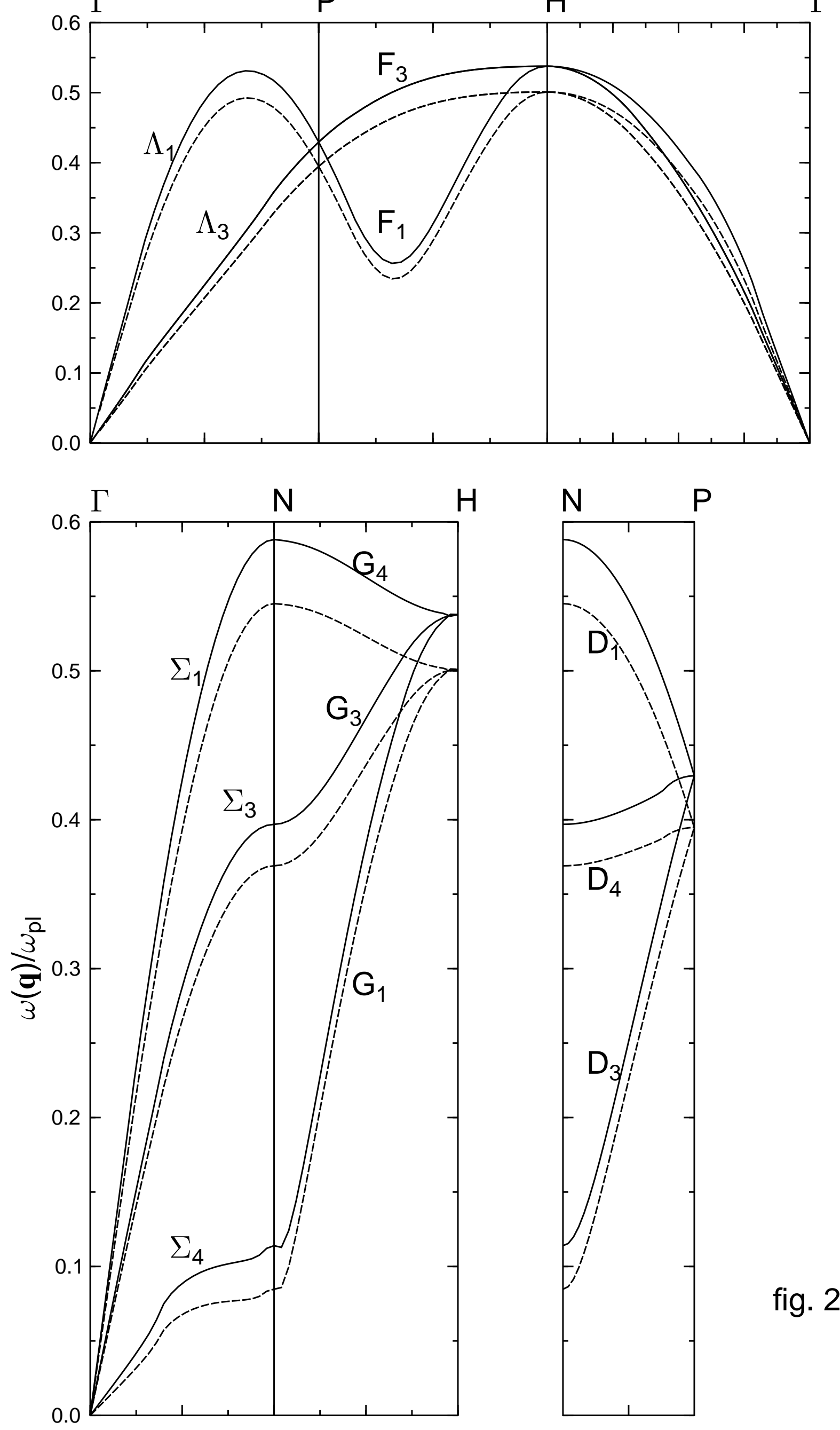

fig. 2 

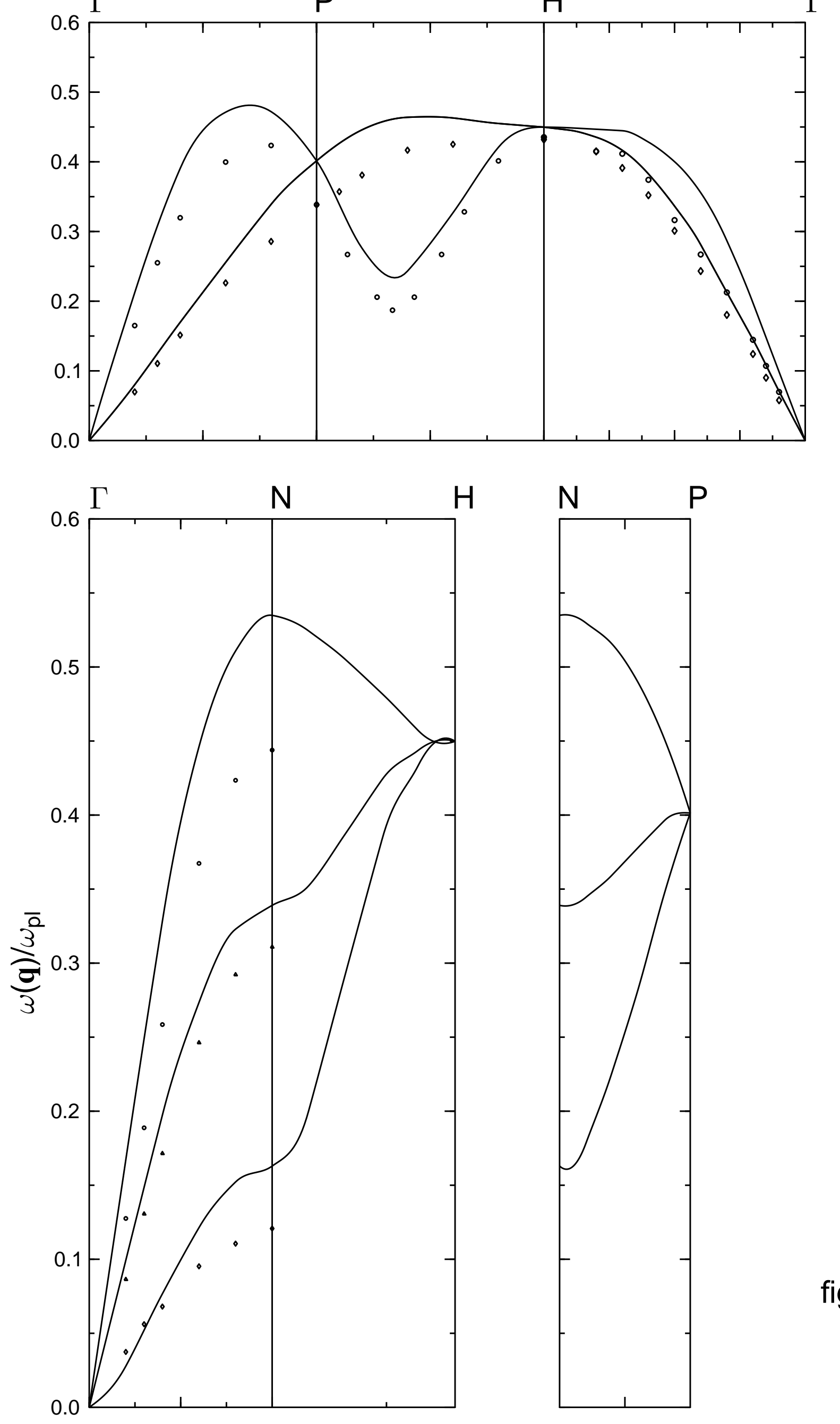

fig. 3 
fig. 4

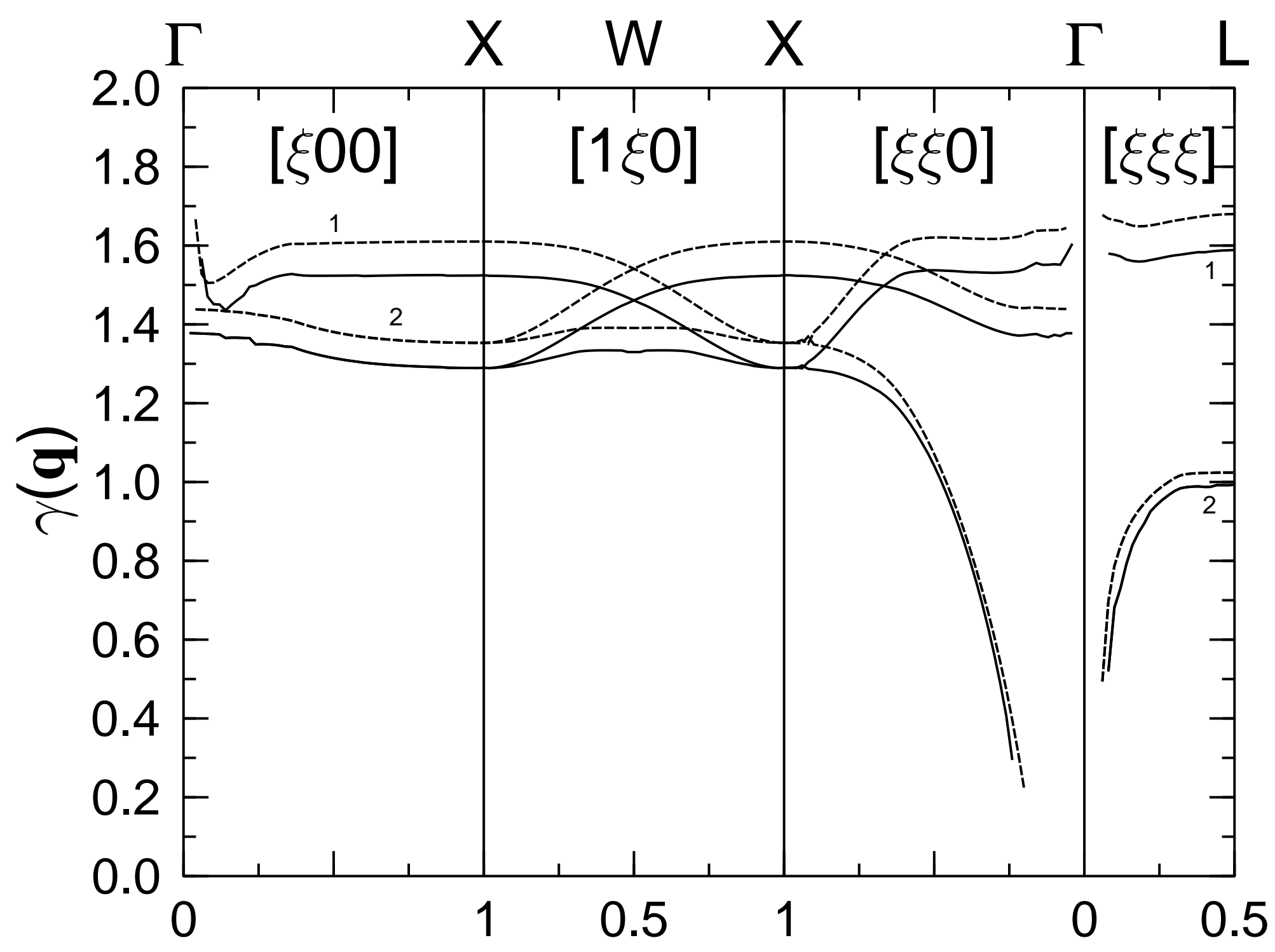


fig. 6

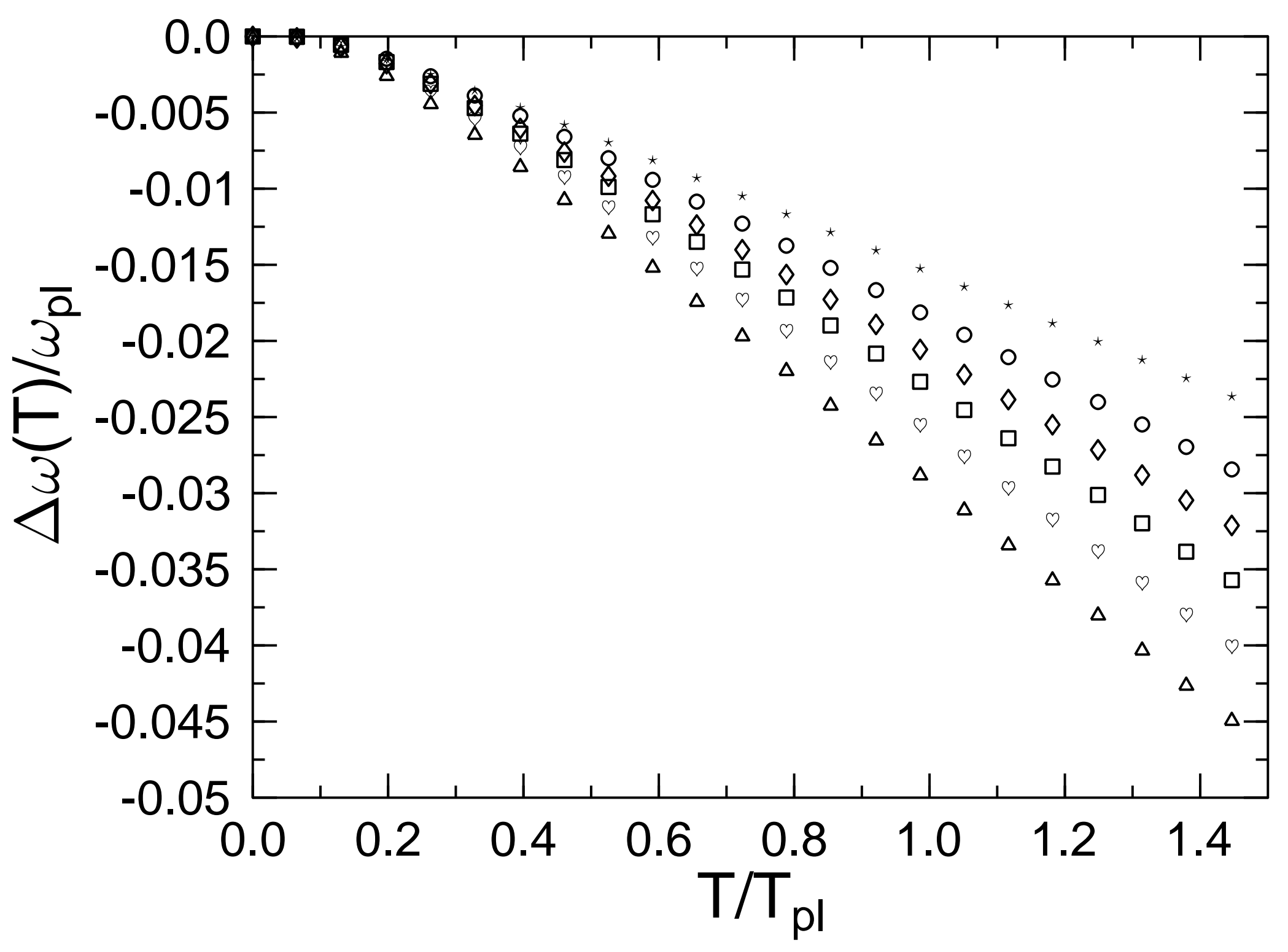


fig. 7

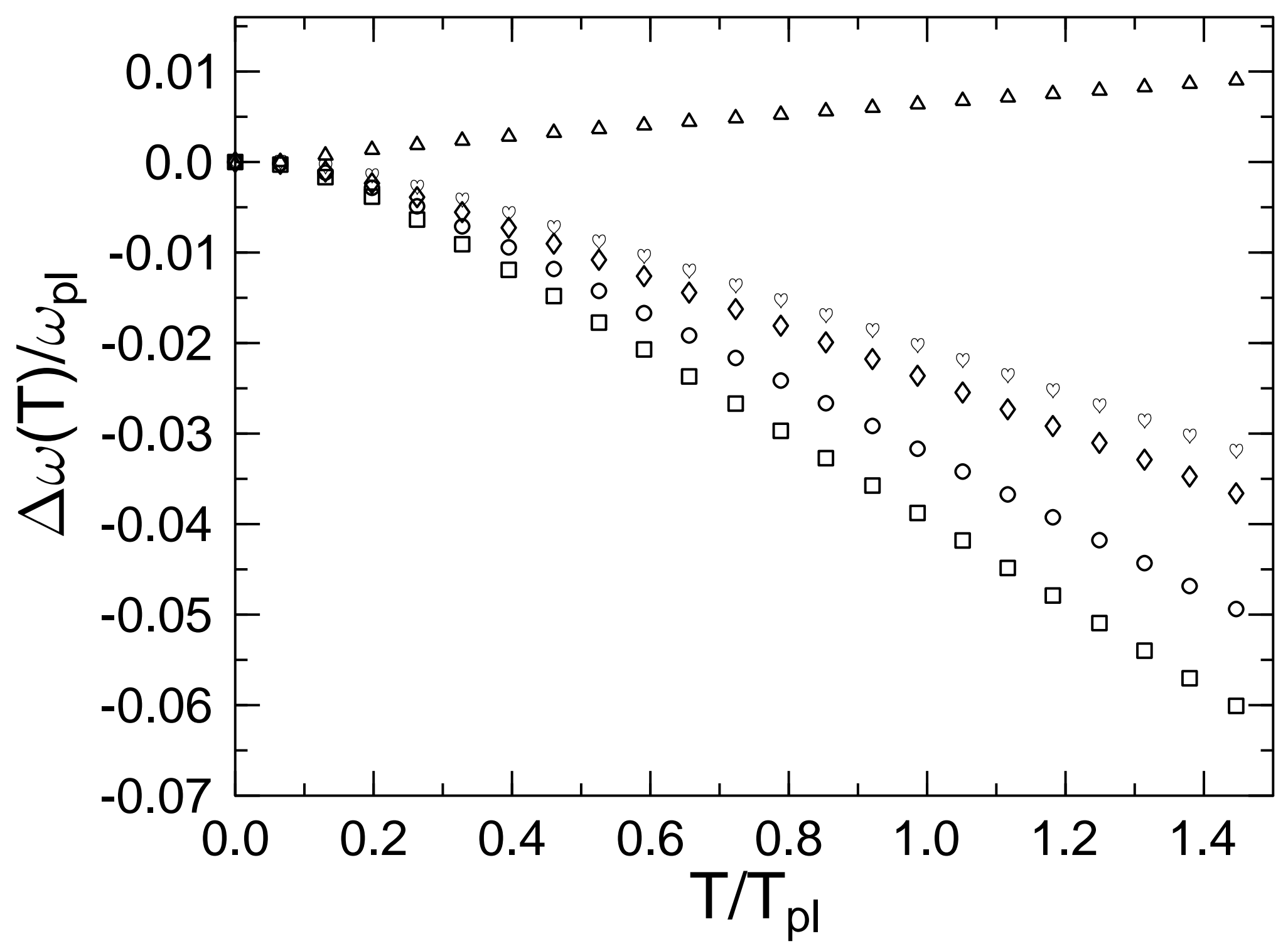


fig. 8

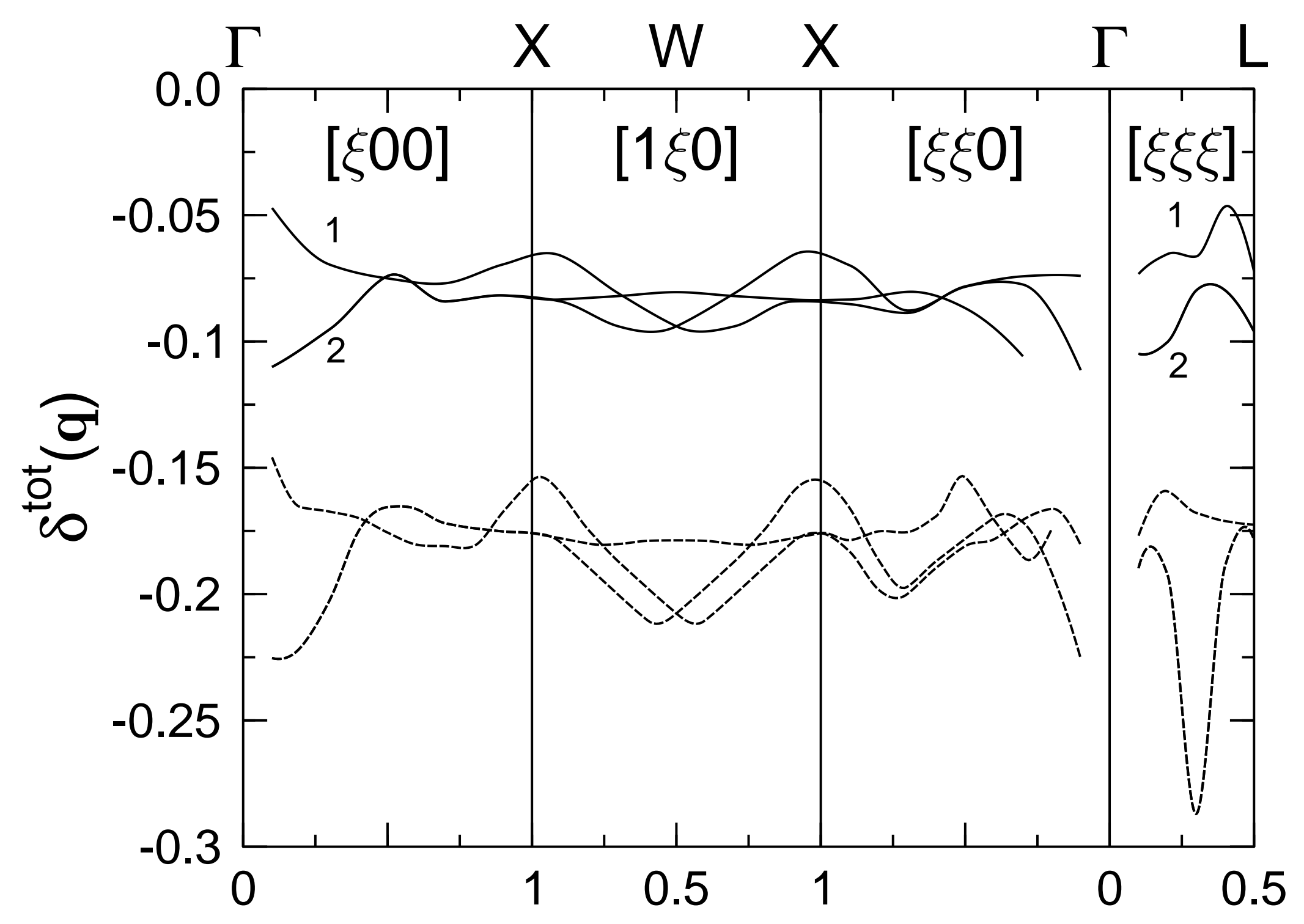




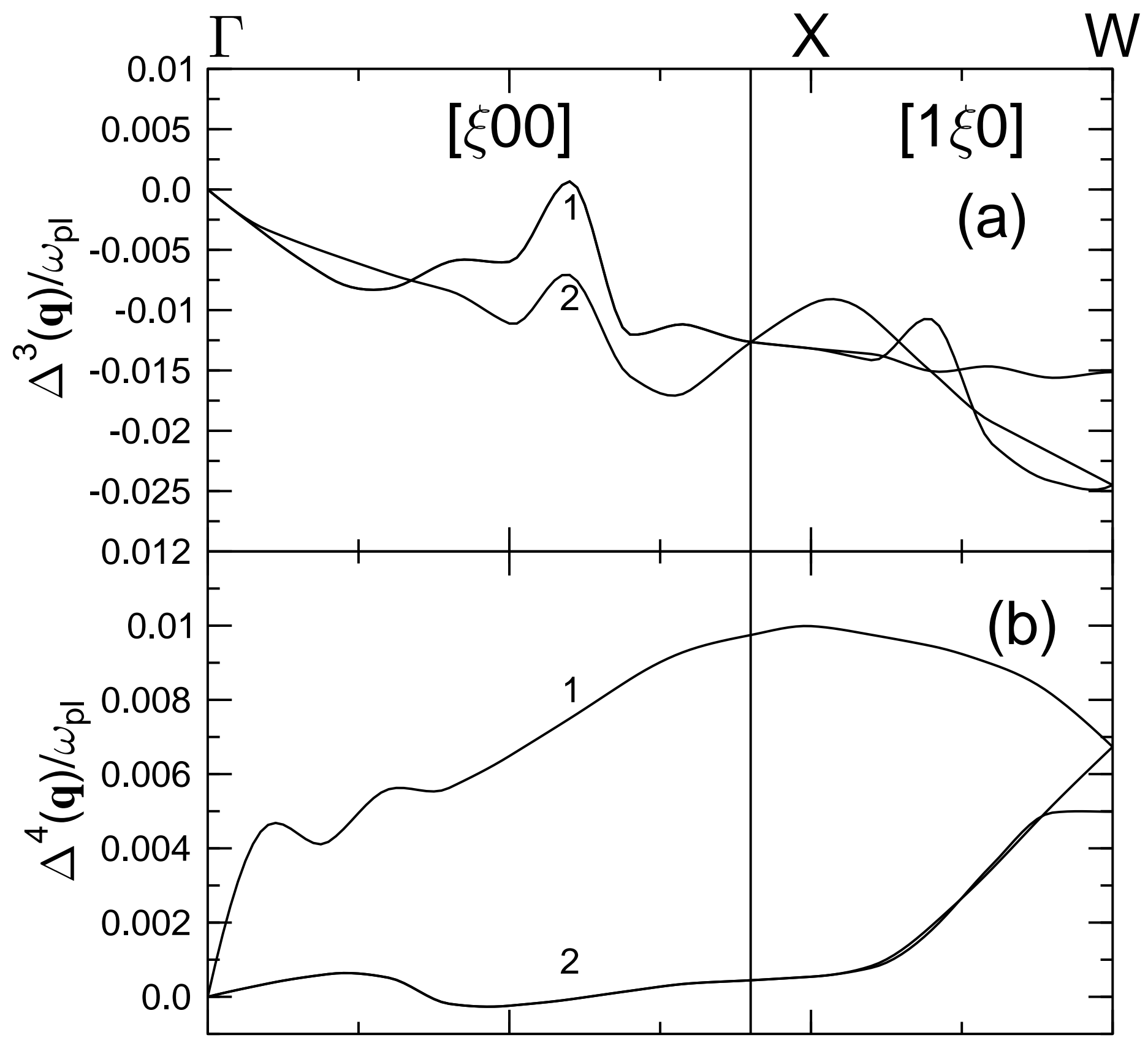

fig. 10 

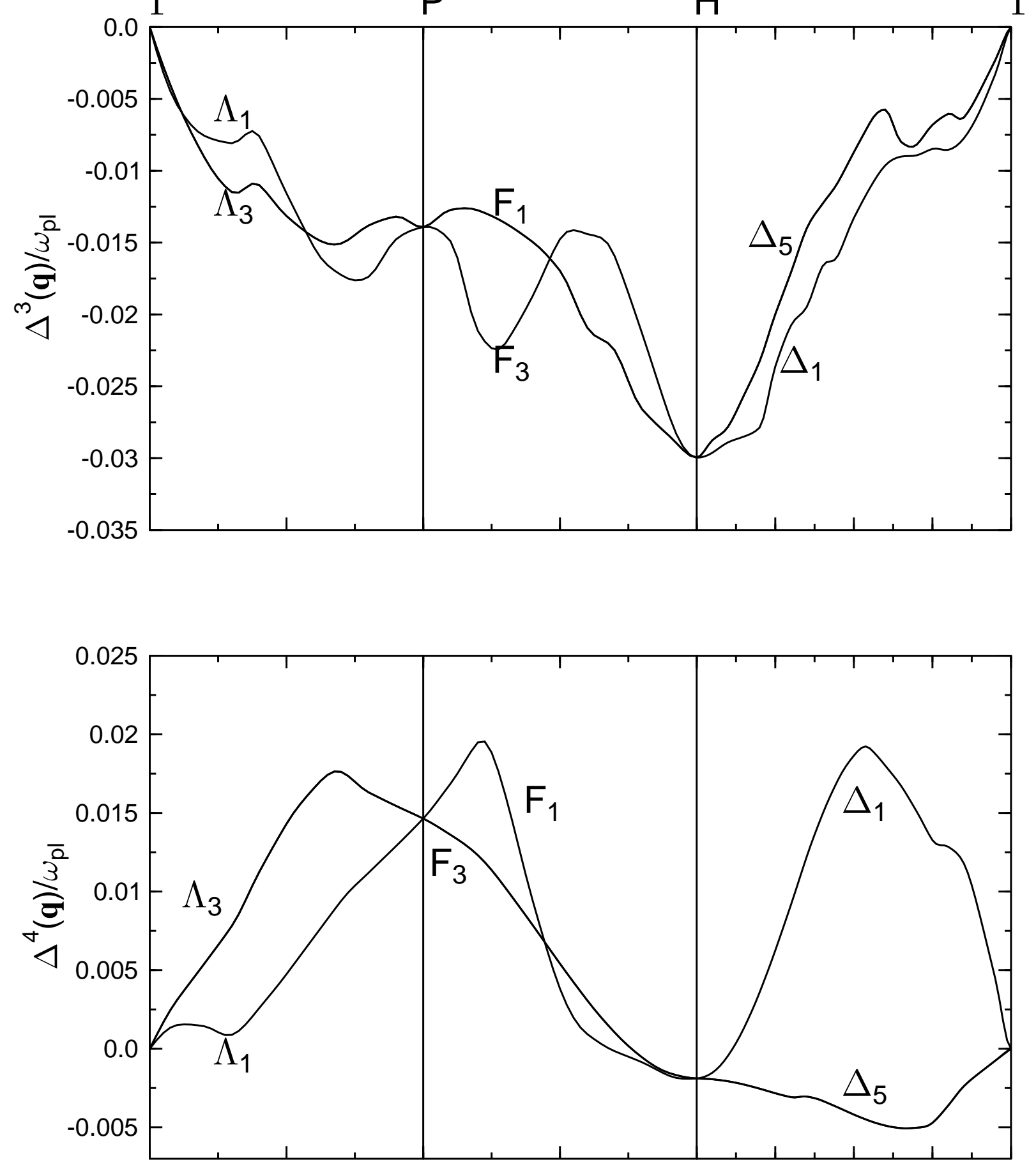

fig. 11 
fig. 12

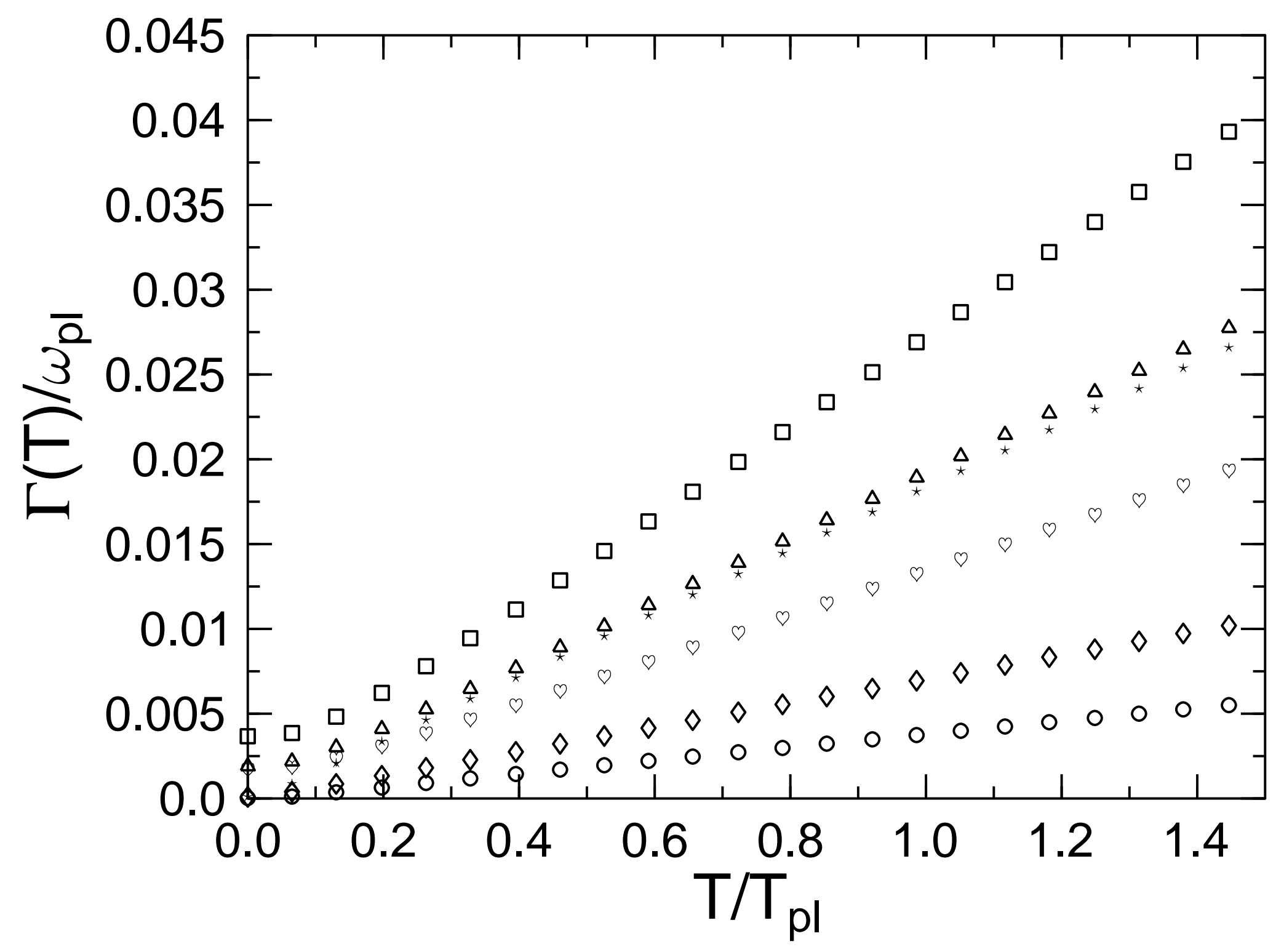


fig. 13

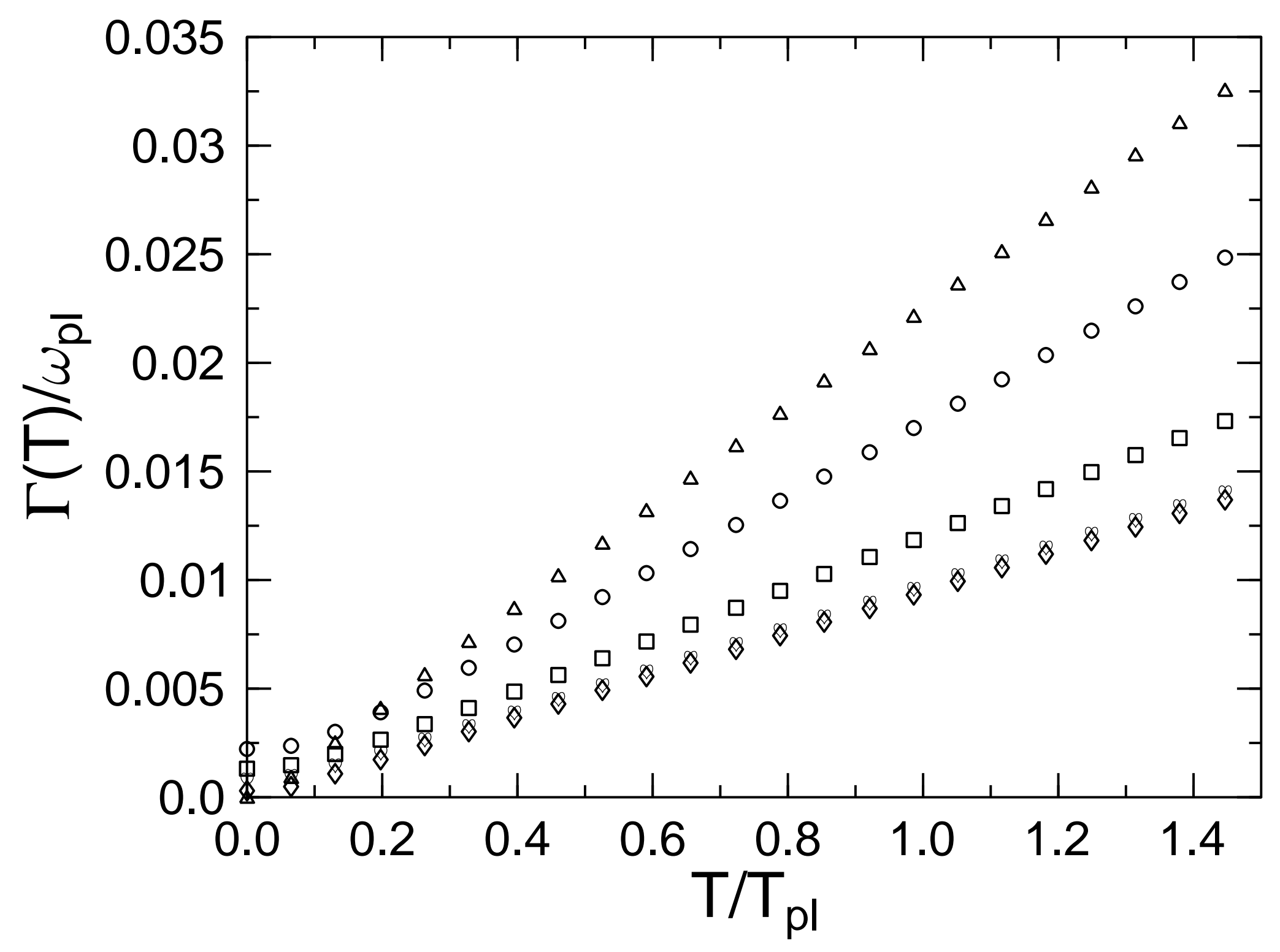


fig. 14

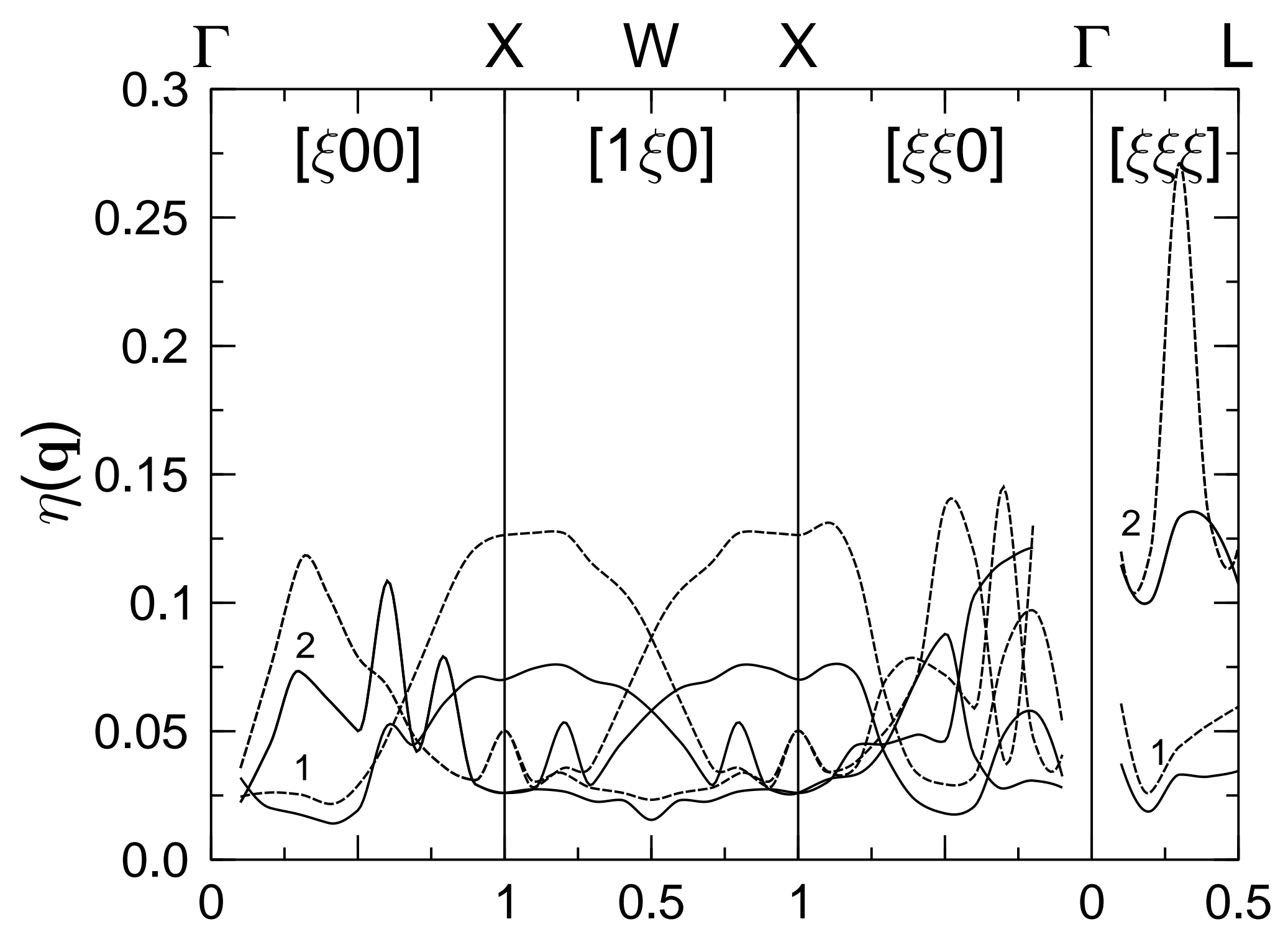


fig. 16

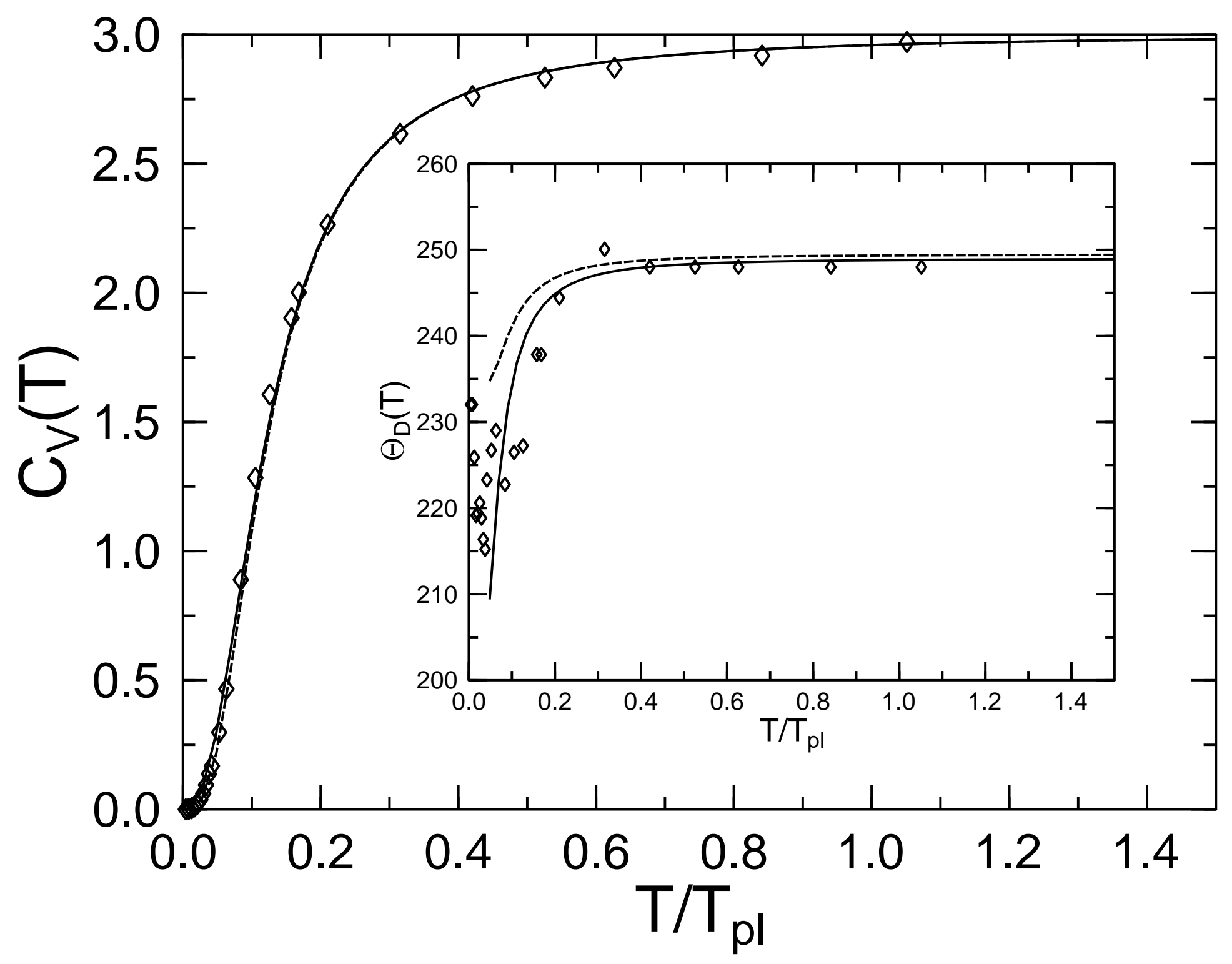


fig. 17

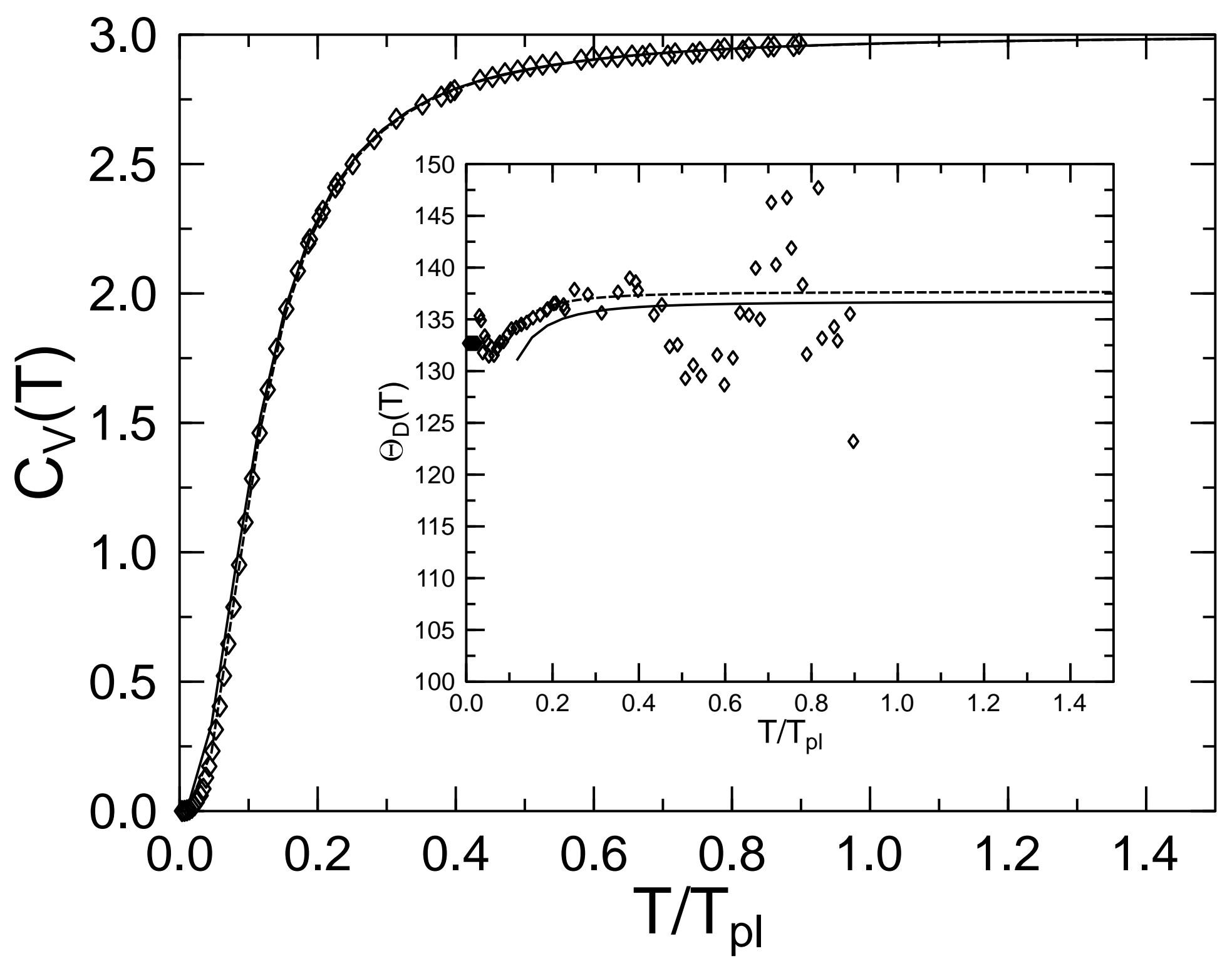


fig. 18

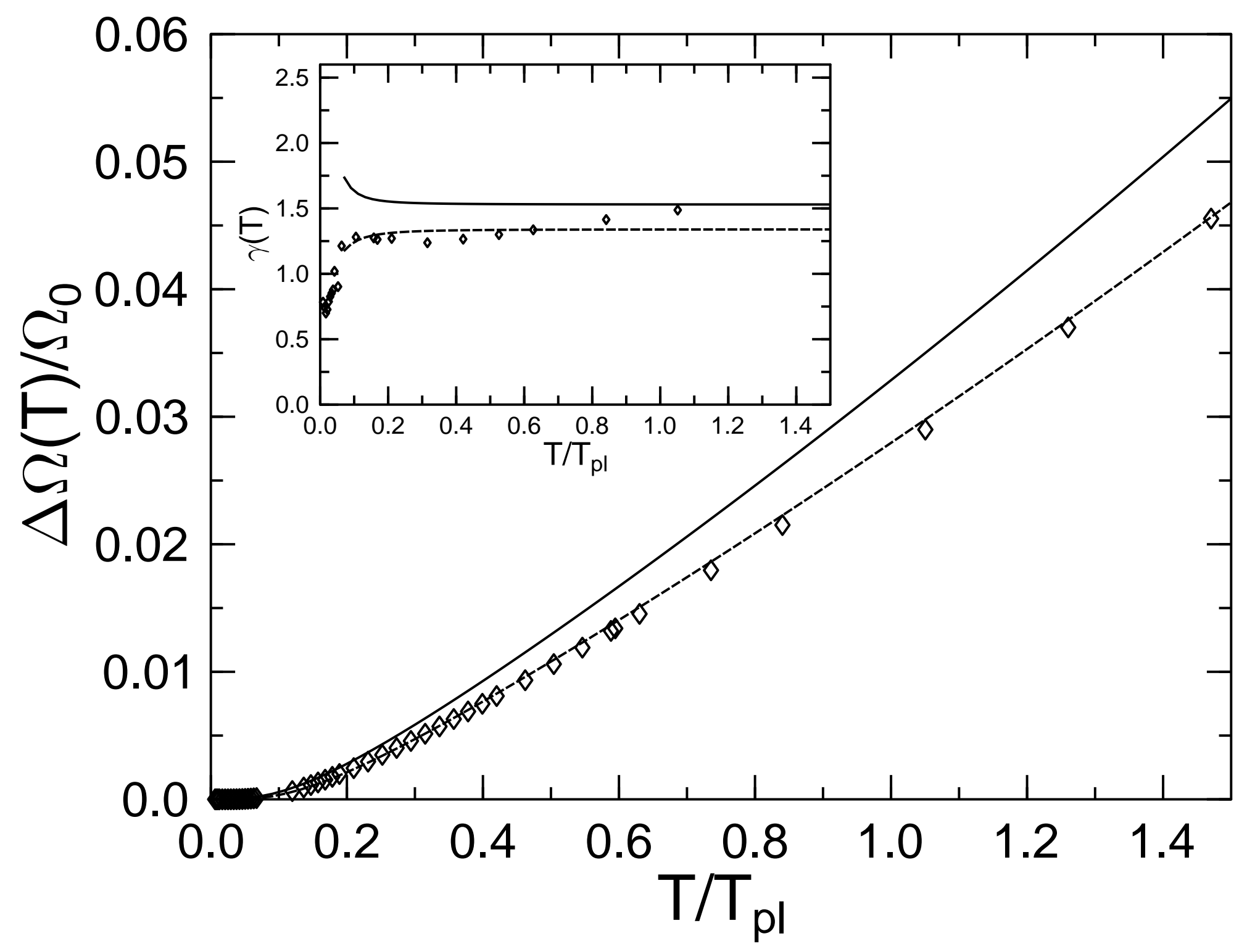


fig. 19

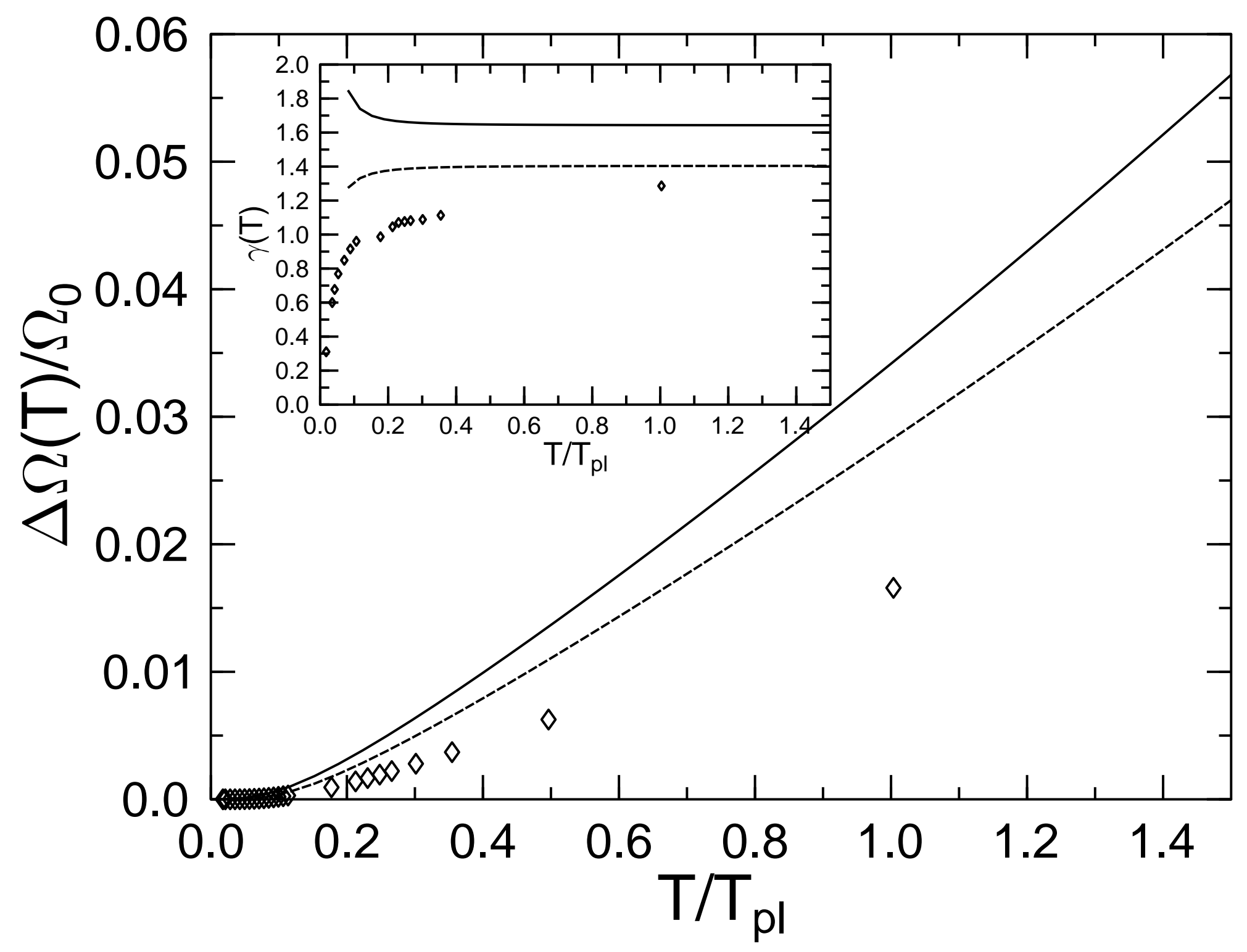

